Topical Review

\title{
Continuous-variable entropic uncertainty relations
}

\author{
Anaelle Hertz ${ }^{1,2}$ and Nicolas $\mathrm{J} \mathrm{Cerf}^{1}$ \\ ${ }^{1}$ Centre for Quantum Information and Communication, École polytechnique de \\ Bruxelles, Université libre de Bruxelles, 1050 Brussels, Belgium
}

E-mail: ahertz@ulb.ac.be and ncerf@ulb.ac.be

Received 4 September 2018, revised 18 January 2019

Accepted for publication 1 February 2019

Published 2 April 2019

\begin{abstract}
Uncertainty relations are central to quantum physics. While they were originally formulated in terms of variances, they have later been successfully expressed with entropies following the advent of Shannon information theory. Here, we review recent results on entropic uncertainty relations involving continuous variables, such as position $x$ and momentum $p$. This includes the generalization to arbitrary (not necessarily canonically-conjugate) variables as well as entropic uncertainty relations that take $x-p$ correlations into account and admit all Gaussian pure states as minimum uncertainty states. We emphasize that these continuous-variable uncertainty relations can be conveniently reformulated in terms of entropy power, a central quantity in the information-theoretic description of random signals, which makes a bridge with variance-based uncertainty relations. In this review, we take the quantum optics viewpoint and consider uncertainties on the amplitude and phase quadratures of the electromagnetic field, which are isomorphic to $x$ and $p$, but the formalism applies to all such variables (and linear combinations thereof) regardless of their physical meaning. Then, in the second part of this paper, we move on to new results and introduce a tighter entropic uncertainty relation for two arbitrary vectors of intercommuting continuous variables that takes correlations into account. It is proven conditionally on reasonable assumptions. Finally, we present some conjectures for new entropic uncertainty relations involving more than two continuous variables.
\end{abstract}

\footnotetext{
${ }^{2}$ Present address: University of Lille, CNRS, UMR 8523—PhLAM—Physique des Lasers Atomes et Molécules, F-59000 Lille, France
} 
Keywords: quantum information, entropic uncertainty relations, continuous variables, quantum optics, Shannon differential entropy, entropy power

(Some figures may appear in colour only in the online journal)

\section{Contents}

1. Introduction

2. Variance-based uncertainty relations 4

2.1. Heisenberg-Kennard uncertainty relation 4

2.2. Schrödinger-Robertson uncertainty relation 5

2.3. Uncertainty relation for more than two observables 7

3. Entropy-based uncertainty relations $\quad 8$

3.1. Shannon differential entropy 8

3.2. Entropy power 9

3.3. Entropic uncertainty relation for canonically conjugate variables 10

3.4. Tight entropic uncertainty relation for canonically conjugate variables 12

3.5. Entropic uncertainty relation for arbitrary quadratures 14

3.6. Other entropic uncertainty relations 16

4. Tight entropic uncertainty relation for arbitrary quadratures 17

4.1. Minimum uncertainty states 17

4.2. Entropic uncertainty relation saturated by all pure Gaussian states 17

4.3. Special case of Gaussian states 19

4.4. Partial proof for all states 20

4.5. Alternative formulation 22

4.6. Entropy-power formulation and covariance-based uncertainty relation $\quad 23$

5. Entropic uncertainty relations for more than two observables 24

6. Conclusion $\quad 29$

Acknowledgments $\quad 29$

Appendix A. Symplectic formalism $\quad 30$

Appendix B. Calculation of $\operatorname{det} \Gamma$

Appendix C. Pure Gaussian states as eigenvectors of $\frac{1}{2} \mathbf{R}^{T} \boldsymbol{\Gamma}^{-1} \mathbf{R}$

References $\quad 36$

\section{Introduction}

The uncertainty principle lies at the heart of quantum physics. It exhibits one of the key discrepancies between a classical and a quantum system. Classically, it is in principle possible to specify the exact value of all measurable quantities in a given state of a system. In contrast, in quantum physics, whenever two observables do not commute, it is impossible to define a quantum state for which their values are simultaneously specified with infinite precision. First expressed by Heisenberg, in 1927, for position and momentum [1] it was formalized by Kennard [2] as

$$
\sigma_{x}^{2} \sigma_{p}^{2} \geqslant \frac{\hbar^{2}}{4}
$$


where $\sigma_{x}^{2}$ and $\sigma_{p}^{2}$ denote the variance of the position $x$ and momentum $p$, respectively, and $\hbar$ is the reduced Planck constant. Shortly after, it was generalized to any pair of observables that do not commute $[3,4]$. The uncertainty principle then states that their values cannot both be sharply defined beyond some precision depending on their commutator.

Aside from variances, another natural way of measuring the uncertainty of a random variable relies on entropy, the central quantity of Shannon information theory. In 1957, Hirschman stated the first entropic uncertainty relation [5] but was only able to prove a weaker form of it. His conjecture was proven in 1975 independently by Bialynicki-Birula and Mycielski [6] and by Beckner [7], making use of the work of Babenko [8]. It reads

$$
h(x)+h(p) \geqslant \ln (\pi e \hbar)
$$

where $h(\cdot)$ is the Shannon differential entropy (see footnote 6 for a dimensionless version of this uncertainty relation). This result is interesting not only because it highlights the fact that Shannon information theory can help better understand fundamental concepts of quantum mechanics, but also because it opened the way to a new and fruitful formulation of uncertainty relations. Why such a success? First because Shannon entropy is arguably the most relevant measure of the degree of randomness (or uncertainty) of a random variable: it measures, in some asymptotic limit, the number of unbiased random bits needed to generate the variable or the number of unbiased random bits that can be extracted from it. In particular, building on Shannon's notion of entropy power, it can easily be seen that the entropic formulation of the uncertainty relation implies Heisenberg relation, so it is somehow stronger [9]. In addition, unlike variance, the entropy is a relevant uncertainty measure even for quantities that are not associated with a numerical value or do not have a natural order. Moreover, entropic uncertainty relations can be generalized in a such way that (nonclassical) correlations with the environment are taken into account: typically, entanglement between a system and its environment can be exploited in order to reduce uncertainty. If an observer has access to a quantum memory, the entropic formulation allows one to establish stronger uncertainty relations, which is particularly useful in quantum key distribution [10, 11]. Uncertainty relations can then be used as a way to verify the security of a cryptographic protocol [12-15]. They also find applications in the context of separability criteria, that is, criteria that enable one to distinguish between entangled and non-entangled states. For example, the positive-partial-transpose separability criterion for continuous variables [16-19] is based on uncertainty relations: it builds on the fact that a state is necessarily entangled if its partial transpose is not physical, which itself is observed by the violation of an uncertainty relation. While [16, 17] use variance-based uncertainty relations for this purpose, $[18,19]$ exploit Shannon differential entropies (separability criteria can also be built with Rényi entropies [20]). In general, a tighter uncertainty relation enables detecting more entangled states, hence finding better uncertainty relations leads to better separability criteria [21].

Somehow surprisingly, although entropic uncertainty relations were first developed with continuous variables, a large body of knowledge has accumulated over years on their discretevariable counterpart. In a seminal work, Deutsch proved in 1983 that $H(A)+H(B)$ has a nontrivial lower bound [22], where $H(\cdot)$ is the Shannon entropy and $A$ and $B$ are two incompatible discrete-spectrum observables. The lower bound was later improved by Kraus [23] and Maassen and Uffink [24], and much work followed on such uncertainty relations, with or without a quantum memory. We refer the reader to the recent review by Coles et al [25], where details on entropic uncertainty relations and their various applications can be found.

There is comparatively less available literature today on continuous-variable entropic uncertainty relations. Beyond [25], the older survey by Bialynicki-Birula and Rudnicki [26] 
focuses on continuous variables but is missing the most recent results, while the recent review by Toscano et al [27] is mainly concerned with coarse-grain measurements, which is a way to bridge the gap between discrete- and continuous-variable systems. With the present paper, we provide an up-to-date overview on continuous-variable entropic uncertainty relations that apply to any pair of canonically conjugate variables and linear combinations thereof. This review is meant to be balanced between the main results on this topic and some of our own recent contributions.

In section 2, we first go over variance-based uncertainty relations as they serve as a reference for the entropic ones. In section 3, we review the properties of Shannon differential entropy as well as the notion of entropy power, and then move on to entropy-based uncertainty relations. In particular, we define the entropic uncertainty relation due to Bialynicki-Birula and Mycielski in section 3.3, and then introduce the entropy-power formulation which we deem appropriate to express continuous-variable uncertainty relations. Sections 3.4 and 3.5 are dedicated to more recent entropic uncertainty relations. In particular, the uncertainty relation of section 3.4 improves the Bialynicki-Birula and Mycielski relation by taking $x$ - $p$ correlations into account, and is then saturated by all pure Gaussian states. The entropic uncertainty relation of section 3.5 is defined for any two vectors of intercommuting continuous variables, which are not necessarily related by a Fourier transform. In section 3.6, we briefly mention other possible variants of entropic uncertainty relations. In the second part of this paper, we move on to new results and present in section 4 a tight entropic uncertainty relation that holds for two vectors of intercommuting continuous variables. This relation is called tight because it is saturated by all pure Gaussian states. Finally, we propose in section 5 several conjectures in order to define an entropic uncertainty relation for more than two variables, and prove one of them. More than two observables have long been considered for variance-based uncertainty relations [28-30], but, to our knowledge, no such result exists yet in terms of continuous entropies (except for a very recent conjecture by Kechrimparis and Weigert [31]).

In appendix A, we give a brief overview on Gaussian states and symplectic transformations, which should help readers who are less familiar with quantum optics to better understand this paper. Appendices B and C provide details on some calculations needed in section 4.

\section{Variance-based uncertainty relations}

\subsection{Heisenberg-Kennard uncertainty relation}

In 1927, Heisenberg first expressed an uncertainty relation between the position and momentum of a particle. In a seminal paper [1], he exhibited a thought experiment - known as the Heisenberg's microscope-for measuring the position of an electron. From this experiment, he concluded that there is a trade-off about how precisely the position $x$ and momentum $p$ can be both measured, which he expressed as $\delta x \delta p \sim h$, where $h$ is the Planck constant. Shortly after, Kennard [2] mathematically formalized the uncertainty relation and proved that

$$
\sigma_{x}^{2} \sigma_{p}^{2} \geqslant \frac{\hbar^{2}}{4}
$$

where $\sigma_{x}^{2}$ and $\sigma_{p}^{2}$ represent the variances of the position and momentum of a quantum particle and $\hbar=h / 2 \pi$ is the reduced Planck constant.

Note that, as expressed by Kennard, the uncertainty relation is actually a property of Fourier transforms. While Heisenberg had made a statement about measurements, Kennard's formulation is really expressing an intrinsic property of the state. Following Heisenberg's view, several papers have focused on finding an appropriate definition for measurement uncertainties 
(see [32] for a review). In particular, Ozawa [33] derived an inequality about error-disturbance and claimed that this is a rigorous version of Heisenberg's formulation of the uncertainty principle. Nevertheless, this claim is still a matter of debate (for more details, see for example $[34,35])$. Nowadays, most textbooks adopt the view of Kennard, as we do here, even though equation (3) is most often called the Heisenberg uncertainty relation.

\subsection{Schrödinger-Robertson uncertainty relation}

The uncertainty relation was originally formulated for position and momentum, but it is well known that it actually holds for any pair of canonically-conjugate variables, i.e. variables related to each other by a Fourier transform. For instance, the (amplitude and phase) quadrature components of a mode of the electromagnetic field are canonically-conjugate variables behaving just as position and momentum ${ }^{3}$. Other canonical pairs can be defined, such as the charge and flux variables in a superconducting Josephson junction, verifying again equation (3). In fact, in 1928, Robertson [36] extended the formulation of the uncertainty principle to any two arbitrary observables $\hat{A}$ and $\hat{B}$ as

$$
\sigma_{A}^{2} \sigma_{B}^{2} \geqslant \frac{1}{4}|\langle\psi|[\hat{A}, \hat{B}]| \psi\rangle|^{2}
$$

where $[\cdot, \cdot]$ stands for the commutator. Obviously, if $\hat{A}=\hat{x}$ and $\hat{B}=\hat{p}$, we recover Heisenberg uncertainty relation since $[\hat{x}, \hat{p}]=\mathrm{i} \hbar$. For simplicity, while being aware that uncertainty relations are expressed in terms of $\hbar$, we now fix $\hbar=1$.

Relation (3) is invariant under $(x, p)$-displacements in phase space since it only depends on central moments (esp. second-order moments of the deviations from the mean). Furthermore, it is saturated by all pure Gaussian states provided that they are squeezed in the $x$ or $p$ direction only. More precisely, if we define the covariance matrix

$$
\gamma=\left(\begin{array}{cc}
\sigma_{x}^{2} & \sigma_{x p} \\
\sigma_{x p} & \sigma_{p}^{2}
\end{array}\right)
$$

where $\gamma_{i j}=\frac{1}{2}\left\langle\left\{\hat{r}_{i}, \hat{r}_{j}\right\}\right\rangle-\left\langle\hat{r}_{i}\right\rangle\left\langle\hat{r}_{j}\right\rangle$ and $\mathbf{r}=(\hat{x}, \hat{p})$, we see that Heisenberg relation is saturated by pure Gaussian states provided the principal axes of $\gamma$ are aligned with the $x$ - and $p$-axes, namely $\sigma_{x p}=0$. The principal axes are defined as the $x_{\theta}$ - and $p_{\theta}$-axes for which $\sigma_{x_{\theta} p_{\theta}}=0$, where

$$
\hat{x}_{\theta}=\cos \theta \hat{x}+\sin \theta \hat{p}, \quad \hat{p}_{\theta}=-\sin \theta \hat{x}+\cos \theta \hat{p}
$$

are obtained by rotating $\hat{x}$ and $\hat{p}$ by an angle $\theta$ as shown in figure 1 .

The fact that equation (3) is saturated only by certain pure Gaussian states is linked to the fact that this uncertainty relation is not invariant under rotations in phase space. The problem of invariance was solved in 1930 by Schrödinger [3] and Robertson [4], who added an anticommutator in relation (4). The improved uncertainty relation for any two arbitrary observables then reads

$$
\sigma_{A}^{2} \sigma_{B}^{2} \geqslant \frac{1}{4}|\langle\{A, B\}\rangle-2\langle A\rangle\langle B\rangle|^{2}+\frac{1}{4}|\langle[A, B]\rangle|^{2}
$$

\footnotetext{
${ }^{3}$ From now on, we consider these quadrature variables, also noted as $x$ and $p$, and do not make a distinction with their spatial counterparts. Thus, we take the quantum optics viewpoint on uncertainty relations and use the symplectic formalism in phase space, see appendix A. We define, for example, uncertainty relations for $n$ modes, while they could address $n$ spatial degrees of freedom as well. Actually, the used formalism throughout the paper is quite general and applies to any canonically-conjugate variables (and linear combination thereof) regardless on their physical meaning.
} 


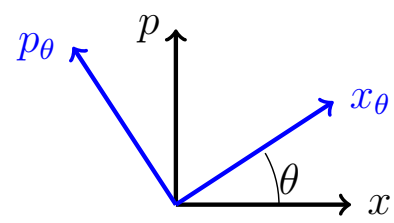

Figure 1. Principal axes $\left(x_{\theta}, p_{\theta}\right)$ of the covariance matrix $\gamma$, defined in such a way that $\sigma_{x_{\theta} p_{\theta}}=0$.

where $\langle\cdot\rangle$ is the shorthand notation for $\langle\psi|\cdot| \psi\rangle$. In the special case of position and momentum, $\hat{A}=\hat{x}$ and $\hat{B}=\hat{p}$, the Robertson-Schrödinger uncertainty relation reads

$$
\operatorname{det} \gamma \geqslant \frac{1}{4}
$$

This uncertainty relation is obviously invariant under symplectic transformations, i.e. squeezing and rotations (see [37] or appendix A for more details on the symplectic formalism and phase-space representation), so it is saturated by all pure Gaussian states, regardless of the orientation of the principal axes of $\gamma$. Indeed, under a symplectic transformation $\mathcal{S}$, the new covariance matrix is given by $\gamma^{\prime}=\mathcal{S} \gamma \mathcal{S}^{T}$. Since the determinant of a symplectic matrix is equal to 1 ,

$$
\operatorname{det} \gamma^{\prime}=\operatorname{det} \mathcal{S} \operatorname{det} \gamma \operatorname{det} \mathcal{S}=\operatorname{det} \gamma
$$

which implies that equation (8) is invariant under symplectic transformations, hence under all Gaussian unitary transformations (since it is also invariant under displacements).

The generalization of the Robertson-Schrödinger uncertainty relation for the position and momentum variables of $n$ modes (or $n$ spatial degrees of freedom) is due to Simon et al [38]. It is formulated as an inequality on the covariance matrix $\gamma$

$$
\gamma+\frac{\mathrm{i}}{2} \Omega \geqslant 0
$$

where

$$
\Omega=\bigoplus_{k=1}^{n} \omega, \quad \omega=\left(\begin{array}{cc}
0 & 1 \\
-1 & 0
\end{array}\right)
$$

For one mode, equation (10) reduces to the Robertson-Schrödinger uncertainty relation, but, in general, we can understand equation (10) as $n$ inequalities that must be satisfied in order for the covariance matrix to represent a physical state. According to Williamson's theorem (see appendix A), we can always diagonalize $\gamma$ in its symplectic form $\gamma^{\oplus}$ with the symplectic values $\nu_{i}$ on the diagonal (each $\nu_{i}$ appearing twice). Therefore, if $\gamma$ is the covariance matrix of a physical state, it satisfies equation (10) and so must $\gamma^{\oplus}$. From this, we can show that equation (10) is equivalent to (see [39] for more details)

$$
\nu_{i} \geqslant \frac{1}{2} \quad \text { for } i=1, \cdots, n \text {. }
$$

Among others, an inequality that is easy to derive from equation (12) is

$$
\operatorname{det} \gamma=\operatorname{det} \gamma^{\oplus}=\prod_{i=1}^{n} \nu_{i}^{2} \geqslant\left(\frac{1}{4}\right)^{n}
$$


which is a straightforward $n$-mode generalization of the Robertson-Schrödinger uncertainty relation.

\subsection{Uncertainty relation for more than two observables}

Before concluding this section, let us mention that, in 1934, Robertson [28] introduced a covariance-based uncertainty relation for $m$ observables which generalizes equation (7). If we define the vector $\mathbf{R}=\left(\hat{R}_{1}, \cdots, \hat{R}_{m}\right)$ of $m$ observables, then the uncertainty relation is expressed as

$$
\operatorname{det} \boldsymbol{\Gamma} \geqslant \operatorname{det} \mathbf{C}
$$

where $\boldsymbol{\Gamma}$ is the covariance matrix of the measured observables and $\mathbf{C}$ the commutator matrix. Their elements are defined as

$$
\Gamma_{i j}=\frac{1}{2}\left\langle\hat{R}_{i} \hat{R}_{j}+\hat{R}_{j} \hat{R}_{i}\right\rangle-\left\langle\hat{R}_{i}\right\rangle\left\langle\hat{R}_{j}\right\rangle, \quad C_{i j}=-\frac{\mathrm{i}}{2}\left\langle\left[\hat{R}_{i}, \hat{R}_{j}\right]\right\rangle,
$$

respectively. For $m=2$, equation (14) reduces to equation (7). Surprisingly, when $m$ is odd, $\operatorname{det} \mathbf{C}=0$. Indeed, $\mathbf{C}$ is an antisymmetric matrix $\left(C_{i j}=-C_{j i}\right)$, so

$$
\mathbf{C}=-\mathbf{C}^{T} \quad \Leftrightarrow \quad \operatorname{det} \mathbf{C}=(-1)^{m} \operatorname{det} \mathbf{C}^{T} \quad \Leftrightarrow \quad \operatorname{det} \mathbf{C}=(-1)^{m} \operatorname{det} \mathbf{C},
$$

which implies that this uncertainty relation is uninteresting for an odd number of observables. For an even number of observables, $\operatorname{det} \mathbf{C}$ is always non-negative [40], so equation (14) is interesting. Note that unlike the situation with the Robertson-Schrödinger uncertainty relation, pure Gaussian states do not, in general, saturate equation (14). For more details on the minimum uncertainty states of this uncertainty relation, see [41].

To circumvent the problem of this irrelevant bound for odd $m$, Kechrimparis and Weigert [29] proved in 2014 that for three pairwise canonical observables defined as $\hat{p}, \hat{x}$ and $\hat{r}=-\hat{x}-\hat{p}$ (which satisfy the commutation relations $[\hat{p}, \hat{x}]=[\hat{x}, \hat{r}]=[\hat{r}, \hat{p}]=-i$ ), the product of variances must satisfy the inequality

$$
\sigma_{x}^{2} \sigma_{p}^{2} \sigma_{r}^{2} \geqslant\left(\frac{1}{\sqrt{3}}\right)^{3}
$$

They later generalized this result to any vector $\mathbf{R}=\left(\hat{R}_{1}, \cdots, \hat{R}_{m}\right)$ of $m$ observables acting on one single mode as [31]

$$
\sigma_{1}^{2} \sigma_{2}^{2} \cdots \sigma_{m}^{2} \geqslant\left(\frac{|\mathbf{a} \wedge \mathbf{b}|}{m}\right)^{m}
$$

where $\sigma_{i}^{2}=\boldsymbol{\Gamma}_{i i}$ are the variances of the $m$ observables, $\mathbf{a}$ and $\mathbf{b}$ are defined through

$$
\mathbf{R}=\mathbf{a} \hat{x}+\mathbf{b} \hat{p}
$$

with $\hat{x}$ and $\hat{p}$ being the canonically conjugate quadratures of the mode, and the square norm of the wedge product $\mathbf{a} \wedge \mathbf{b}$ is computed as

$$
|\mathbf{a} \wedge \mathbf{b}|^{2}=\sum_{i>j=1}^{m}\left(a_{i} b_{j}-a_{j} b_{i}\right)^{2}=|\mathbf{a}|^{2}|\mathbf{b}|^{2}-(\mathbf{a} \cdot \mathbf{b})^{2} .
$$

Shortly after, Dodonov also derived a general uncertainty relation involving any triple or quadruple of observables [30]. 
Note that equation (18) takes a simple form in the special case where the $m$ one-modal observables are equidistributed quadratures over the unit circle, that is

$$
\hat{R}_{i}=\cos \phi_{i} \hat{x}+\sin \phi_{i} \hat{p} \quad \text { with } \quad \phi_{i}=\frac{2 \pi(i-1)}{m}, \quad i=1, \ldots, m .
$$

Indeed, the square norm of the wedge product $\mathbf{a} \wedge \mathbf{b}$ may be related to the matrix of commutators $\mathbf{C}$ as

$$
|\mathbf{a} \wedge \mathbf{b}|^{2}=4 \sum_{i>j=1}^{m}\left|C_{i j}\right|^{2}
$$

where the $C_{i j}$ are defined in equation (15). Then, for the observables $\hat{R}_{i}$ of equation (21), it can be shown that

$$
C_{i j}=\frac{1}{2} \sin \left(\frac{2 \pi}{m}(j-i)\right)
$$

so that

$$
|\mathbf{a} \wedge \mathbf{b}|^{2}=\sum_{i>j=1}^{m} \sin ^{2}\left(\frac{2 \pi}{m}(j-i)\right)=\frac{m^{2}}{4} .
$$

Plugging this into equation (18) leads to the uncertainty relation [31]

$$
\sigma_{1}^{2} \sigma_{2}^{2} \cdots \sigma_{m}^{2} \geqslant\left(\frac{1}{2}\right)^{m}
$$

\section{Entropy-based uncertainty relations}

\subsection{Shannon differential entropy}

We start by reviewing the main properties of Shannon differential (continuous-variable) entropy. The differential entropy of a continuous (i.e. real-valued) variable $X$ with probability distribution $p(x)$ measures its uncertainty and is defined as

$$
h(X) \equiv h[p]=-\int_{-\infty}^{\infty} \mathrm{d} x p(x) \ln p(x) .
$$

Here, the notation $h[p]$ implies that the entropy is a functional of the probability distribution $p(x)$, but it is often written $h(X)$ to stress that it refers to the random variable $X$. The definition (26) of the differential entropy is the natural continuous extension of the discrete entropy. More precisely, $h(X)$ is the limit of $H\left(X^{\Delta}\right)+\log \Delta$ when $\Delta \rightarrow 0$, where $H\left(X^{\Delta}\right)$ is the discrete entropy of $X^{\Delta}$ defined as the discretized version of variable $X$ with discretization step $\Delta$. More details can be found in [26, 42].

For the probability distribution $p\left(x_{1}, \cdots, x_{m}\right)$ of $m$ continuous variables, we define the joint differential entropy of the vector $\mathbf{X}=\left(X_{1}, \cdots, X_{m}\right)$ as

$$
h(\mathbf{X})=-\int \mathrm{d} x_{1} \cdots \mathrm{d} x_{m} p\left(x_{1}, \cdots, x_{m}\right) \ln p\left(x_{1}, \cdots, x_{m}\right) .
$$

In addition, just like for discrete entropies, we may define the mutual information between two continuous variables $X_{1}$ and $X_{2}$ as 


$$
I\left(X_{1}: X_{2}\right)=h\left(X_{1}\right)+h\left(X_{2}\right)-h\left(X_{1}, X_{2}\right)
$$

where $h\left(X_{1}, X_{2}\right)$ is the joint differential entropy and $h\left(X_{1}\right)$ and $h\left(X_{2}\right)$ are the differential entropies of the two marginals. The mutual information measures the shared entropy between $X_{1}$ and $X_{2}$ and is always non-negative.

Let us mention some useful properties of the differential entropy [42]:

- The differential entropy can be negative (unlike the discrete-variable entropy).

- The differential entropy is concave in $p(x)$.

- The differential entropy is subadditive

$$
h(\mathbf{X}) \leqslant \sum_{i} h\left(X_{i}\right)
$$

- Under a translation, the value of the differential entropy does not change

$$
h(\mathbf{X}+\mathbf{c})=h(\mathbf{X}),
$$

where $\mathbf{c}$ is an arbitrary real vector.

- Under a linear transformation, the differential entropy changes as

$$
h(A \mathbf{X})=h(\mathbf{X})+\ln |\operatorname{det} A|,
$$

where $A$ is an invertible matrix that transforms the vector $\mathbf{X}$.

Note that the Shannon differential entropy actually belongs to the larger family of Rényi entropies. The Rényi entropy $h_{\alpha}(X)$ of parameter $\alpha$ is defined as

$$
h_{\alpha}(X)=\frac{1}{1-\alpha} \log \left[\int_{-\infty}^{\infty} \mathrm{d} x p^{\alpha}(x)\right]
$$

and the limit of this expression when $\alpha \rightarrow 1$ converges to Shannon entropy, namely $\lim _{\alpha \rightarrow 1} h_{\alpha}(X)=h(X)$. Properties (30) and (31) still hold for Rényi entropies, while it is not the case for concavity and subadditivity.

\subsection{Entropy power}

Of particular interest is the entropy of a Gaussian distribution. Let $\mathbf{X}=\left(X_{1}, \cdots, X_{m}\right)$ be a vector of $m$ Gaussian-distributed (possibly correlated) variables,

$$
p_{\mathrm{G}}(\mathbf{x})=\frac{1}{\sqrt{(2 \pi)^{m} \operatorname{det} \gamma}} \mathrm{e}^{-\frac{1}{2}(\mathbf{x}-\langle\mathbf{x}\rangle)^{T} \gamma^{-1}(\mathbf{x}-\langle\mathbf{x}\rangle)}
$$

where $\mathbf{x}=\left(x_{1} \cdots, x_{m}\right)^{T}$ and $\gamma$ is the covariance matrix. Its entropy is given by

$$
h(\mathbf{X})=\frac{1}{2} \ln \left((2 \pi e)^{m} \operatorname{det} \gamma\right)
$$

For two Gaussian variables $X_{1}$ and $X_{2}$, the mutual information is given by

$$
I_{\mathrm{G}}\left(X_{1}: X_{2}\right)=\frac{1}{2} \ln \left(\frac{\sigma_{1}^{2} \sigma_{2}^{2}}{\operatorname{det} \gamma}\right)
$$

where $\sigma_{i}^{2}$ is the variance of $X_{i}(i=1,2)$ and $\gamma$ is the covariance matrix of variables $X_{1}$ and $X_{2}$.

A key property of Gaussian distributions is that among all distributions $p(\mathbf{x})$ with a same covariance matrix $\gamma$, the one having the maximum entropy is the Gaussian distribution $p_{\mathrm{G}}(\mathbf{x})$, that is 


$$
h[p] \leqslant h\left[p_{\mathrm{G}}\right]=\frac{1}{2} \ln \left((2 \pi e)^{m} \operatorname{det} \gamma\right) .
$$

Note that the equality is reached if and only if $p(\mathbf{x})$ is Gaussian.

From the subadditivity of the entropy applied to a multivariate Gaussian distribution, we get the Hadamard inequality

$$
\operatorname{det} \gamma \leqslant \prod_{i}^{m} \sigma_{i}^{2},
$$

from which we can derive

$$
\sigma_{1}^{2} \sigma_{2}^{2} \cdots \sigma_{m}^{2} \geqslant \operatorname{det} \mathbf{C}
$$

which is a weaker form of Robertson uncertainty relation (14) for $m$ observables that ignores the correlations between them.

Now, exploiting (34), we define the entropy power of a set of $m$ continuous random variables $\mathbf{X}=\left(X_{1}, \cdots, X_{m}\right)$ as

$$
N_{\mathbf{x}}=\frac{1}{2 \pi e} \mathrm{e}^{\frac{2}{m} h(\mathbf{x})}
$$

It is the variance ${ }^{4}$ of a set of $m$ independent Gaussian variables that produce the same entropy as the set $\mathbf{X}$. The fact that the maximum entropy is given by a Gaussian distribution for a fixed covariance matrix $\gamma$ translates, in terms of entropy powers, to

$$
N_{\mathbf{x}} \leqslant(\operatorname{det} \gamma)^{1 / m}
$$

For one variable, the entropy power is upper bounded simply by the variance, that is, $N_{x} \leqslant \sigma_{x}^{2}$. In the next section, we will show that the entropy power is a relevant quantity in order to express entropic uncertainty relations [9].

\subsection{Entropic uncertainty relation for canonically conjugate variables}

The first formulation of an uncertainty relation in terms of entropies is due to Hirschman [5] in 1957. He conjectured an entropic uncertainty relation (EUR) for the position and momentum observables, which reads as follows:

EUR for canonically-conjugate variables [6, 7]:

Any $n$-modal state $\rho$ satisfies the entropic uncertainty relation

$$
h(\mathbf{x})+h(\mathbf{p}) \geqslant n \ln (\pi e \hbar)
$$

where $\mathbf{x}=\left(\hat{x}_{1}, \cdots, \hat{x}_{n}\right)$ and $\mathbf{p}=\left(\hat{p}_{1}, \cdots, \hat{p}_{n}\right)$ are two vectors of pairwise canonically-conjugate quadratures ${ }^{5}$ and $h(\cdot)$ is the differential entropy defined in equation (27).

Hirschman was only able to prove a weaker form of this conjecture (where $e$ is replaced by 2 in the lower bound) because of the known bound in the Hausdorff-Young inequality at the time. The Hausdorff-Young inequality, which applies to Fourier transforms, is indeed at the heart of the proof of entropic uncertainty relations for canonically conjugate variables. A

\footnotetext{
${ }^{4}$ Although it is a variance, it is called 'power' as it was introduced by Shannon in the context of the informationtheoretic description of time-dependent signals.

${ }^{5}$ From now on, we make no precise distinction between the quadrature $\hat{x}(\hat{p})$ and the random variable $X(P)$ that results from its measurement. The entropies of the random variables $X$ and $P$ will thus be noted $h(\hat{x})$ and $h(\hat{p})$, or simply $h(x)$ and $h(p)$
} 
better bound was later found by Babenko [8] in 1961 and then by Beckner [43] in 1975 (see also the work of Brascamp and Lieb [44]). This led to what is called the Babenko-Beckner inequality for Fourier transforms,

$$
\left(\int \mathrm{d} \mathbf{x}|\mathcal{F} f(\mathbf{x})|^{p}\right)^{1 / p} \leqslant k(p, q)\left(\int \mathrm{d} \mathbf{x}|f(\mathbf{x})|^{q}\right)^{1 / q}
$$

where $\frac{1}{p}+\frac{1}{q}=1, k(p, q)=\left(\frac{2 \pi}{p}\right)^{n / 2 p}\left(\frac{2 \pi}{q}\right)^{-n / 2 q}$ and $\mathcal{F} f$ is the Fourier transform of function $f$. Using this last inequality, Bialynicki-Birula and Mycielski [6] and independently Beckner [7] finally proved equation (41) in 1975.

Let us point out that equation (41) may look weird at first sight as we take the logarithm of a quantity with dimension $\hbar$. This is a feature of the differential entropy itself since we have a similar issue in its definition, equation (26), but the problem actually cancels out in equation (41) since we have dimension $\hbar$ on both sides of the inequality ${ }^{6}$. More rigorously, equation (41) may be understood as the limit of a discretized version of the entropic uncertainty relation, with a discretization step tending to zero [26]. Being aware of this slight abuse of notation, we now prefer to keep $\hbar=1$ for simplicity.

As mentioned in [6], an interesting feature of inequality (41) is that it is stronger thanhence it implies-Heisenberg uncertainty relation, equation (3). This is easy to see if we formulate equation (41) in terms of entropy powers for one mode. Indeed, using equation (39), the entropy powers of $x$ and $p$ are defined as

$$
N_{x}=\frac{1}{2 \pi e} \mathrm{e}^{2 h(x)}, \quad N_{p}=\frac{1}{2 \pi e} \mathrm{e}^{2 h(p)},
$$

so that the entropic uncertainty relation for one mode can be rewritten in the form of an entropy-power uncertainty relation [9]

$$
N_{x} N_{p} \geqslant \frac{1}{4}
$$

which closely resembles the Heisenberg relation (3) with $\hbar=1$. Since $N_{x} \leqslant \sigma_{x}^{2}$ and $N_{p} \leqslant \sigma_{p}^{2}$, which reflects the fact that the Gaussian distribution maximizes the entropy for a fixed variance, we get the chain of inequalities

$$
\sigma_{x}^{2} \sigma_{p}^{2} \geqslant N_{x} N_{p} \geqslant \frac{1}{4}
$$

so that equation (44) implies the Heisenberg relation $\sigma_{x}^{2} \sigma_{p}^{2} \geqslant 1 / 4$. Note that since $N_{x}=\sigma_{x}^{2}$ $\left(N_{p}=\sigma_{p}^{2}\right)$ if and only if $x(p)$ has a Gaussian distribution, the entropic uncertainty relation is strictly stronger than the Heisenberg relation for non-Gaussian states. As emphasized by Son [45], the entropic uncertainty relation may indeed be viewed as an improved version of the Heisenberg relation where the lower bound is lifted up by exploiting an entropic measure of the non-Gaussianity of the state [46], namely

$$
\sigma_{x}^{2} \sigma_{p}^{2} \geqslant \frac{1}{4} \mathrm{e}^{2 D\left(x|| x_{\mathrm{G}}\right)+2 D\left(p \| p_{\mathrm{G}}\right)}
$$

where $D\left(x \| x_{\mathrm{G}}\right)=h\left(x_{G}\right)-h(x) \geqslant 0$ (and similarly for $p$ ) is the relative entropy between $x$ and $x_{\mathrm{G}}$, namely the Gaussian-distributed variable with the same variance as $x$.

\footnotetext{
${ }^{6}$ This problem was absent in the original expression of this uncertainty relation [6] because the variable $k=p / \hbar$ was considered instead of $p$, giving $h(x)+h(k) \geqslant \ln (\pi e)$ for $n=1$.
} 
Just as the Heisenberg uncertainty relation, the entropy-power uncertainty relation (44) is only saturated for pure Gaussian states whose $\gamma$ has principal axes aligned with the $x$ - and $p$-axes (i.e. $\sigma_{x p}=0$ ). It suggests that there is room for a tighter entropic uncertainty relation that is saturated for all pure Gaussian states, in analogy with the Robertson-Schrödinger uncertainty relation (8). This is the topic of section 3.4.

As a final note, let us mention that one can also write an uncertainty relation for Rényi entropies as defined in equation (32). It reads as follows:

Rényi EUR for canonically-conjugate variables [47]:

Any $n$-modal state $\rho$ satisfies the entropic uncertainty relation

$$
h_{\alpha}(\mathbf{x})+h_{\beta}(\mathbf{p}) \geqslant n \ln (\pi)+\frac{n \ln (\alpha)}{2(\alpha-1)}+\frac{n \ln (\beta)}{2(\beta-1)}
$$

where $\mathbf{x}=\left(\hat{x}_{1}, \cdots, \hat{x}_{n}\right)$ and $\mathbf{p}=\left(\hat{p}_{1}, \cdots, \hat{p}_{n}\right)$ are two vectors of pairwise canonically-conjugate quadratures and $h_{\alpha}(\cdot)$ is the Rényi entropy defined in equation (32), with parameters $\alpha$ and $\beta$ satisfying

$$
\frac{1}{\alpha}+\frac{1}{\beta}=2 \text {. }
$$

In [48], the entropy-power formulation associated with Rényi entropies was used to show that some Gaussian states saturate these entropic uncertainty relations for all parameter $\alpha$ and $\beta$ such that $\frac{1}{\alpha}+\frac{1}{\beta}=2$. However, for some parameters, it is possible to find non-Gaussian states that saturate them too. For more information about entropic uncertainty relations with Rényi entropies, see also [49-51].

\subsection{Tight entropic uncertainty relation for canonically conjugate variables}

The entropic uncertainty relation, equation (41), is not invariant under all symplectic transformations and is not saturated by all pure Gaussian states. However, a tighter entropic uncertainty relation can be written, which, by taking correlations into account, becomes saturated for all Gaussian pure states. It is expressed as follows.

Tight EUR for canonically-conjugate variables [9]: Any $n$-modal state $\rho$ satisfies the entropic uncertainty relation ${ }^{7}$

$$
h(\mathbf{x})+h(\mathbf{p})-\frac{1}{2} \ln \left(\frac{\operatorname{det} \gamma_{\mathbf{x}} \operatorname{det} \gamma_{\mathbf{p}}}{\operatorname{det} \gamma}\right) \geqslant n \ln (\pi e)
$$

where $\mathbf{x}=\left(\hat{x}_{1}, \cdots, \hat{x}_{n}\right)$ and $\mathbf{p}=\left(\hat{p}_{1}, \cdots, \hat{p}_{n}\right)$ are two vectors of pairwise canonically-conjugate quadratures and $h(\cdot)$ is the differential entropy defined in equation (27). The covariance matrix $\gamma$ is defined as $\gamma_{i j}=\operatorname{Tr}\left[\hat{\rho}\left\{r_{i}, r_{j}\right\}\right] / 2-\operatorname{Tr}\left[\hat{\rho} r_{i}\right] \operatorname{Tr}\left[\hat{\rho} r_{j}\right]$, with $\mathbf{r}=\left(\hat{x}_{1}, \ldots, \hat{x}_{n}, \hat{p}_{1}, \ldots \hat{p}_{n}\right)$, and $\gamma_{x}\left(\gamma_{p}\right)$ denotes the reduced covariance matrix of the $\mathbf{x}(\mathbf{p})$ quadratures. Equation (49) is saturated if and only if $\rho$ is Gaussian and pure.

In the context of entropic uncertainty relations, it would be natural to take correlations into account via the joint entropy $h(x, p)$ of the two canonically-conjugate quadratures $\hat{x}$ and $\hat{p}$ (considering the case of a single mode, $n=1$ ). The problem, however, is that $h(x, p)$ is not defined for states with a negative Wigner function (more details can be found in $[9,39]$ ). To overcome this problem, correlations can be accounted for by exploiting the covariance

\footnotetext{
${ }^{7}$ The proof is conditional on two reasonable assumptions, see below and [9].
} 
matrix $\gamma$. Indeed, the mutual information $I(x: p)$ between two Gaussian variables $(x$ and $p$ for a one-modal state) can be expressed in terms of the covariance matrix, see equation (35). Then, starting from the joint entropy $h(x, p)=h(x)+h(p)-I(x: p)$ and substituting $I(x: p)$ by its Gaussian form, equation (35), we get a quantity that is defined for all states regardless of whether the Wigner function is positive or not. This yields a tight entropic uncertainty relation [9]

$$
h(x)+h(p)-\frac{1}{2} \ln \left(\frac{\sigma_{x}^{2} \sigma_{p}^{2}}{\operatorname{det} \gamma}\right) \geqslant \ln (\pi e)
$$

whose generalization to $n$ modes corresponds to equation (49). Thus, the lower bound of the entropic uncertainty relation (41) can be lifted up by a non-negative term that exploits the covariance matrix $\gamma$.

The entropic uncertainty relation (49) applies to any state, Gaussian or not. For Gaussian states, it is easy to prove. Indeed, using equation (34) and det $\gamma \geqslant 1 / 4^{n}$ (which is simply the $n$-modal version of Robertson-Schrödinger uncertainty relation, equation (13)), we have

$$
\begin{aligned}
h(\mathbf{x})+h(\mathbf{p})-\frac{1}{2} \ln \left(\frac{\operatorname{det} \gamma_{\mathbf{x}} \operatorname{det} \gamma_{\mathbf{p}}}{\operatorname{det} \gamma}\right) & =n \ln (\pi e)+\frac{1}{2} \ln \left(4^{n} \operatorname{det} \gamma\right) \\
& \geqslant n \ln (\pi e) .
\end{aligned}
$$

This inequality is saturated if and only if the state is pure since $\operatorname{det} \gamma=1 / 4^{n}$ for pure Gaussian states only. Thus, equation (49) is a tight uncertainty relation in the sense that it is saturated for all pure Gaussian states, regardless of the orientation of the principal axes. Nevertheless, equation (49) is not invariant under rotations.

For non-Gaussian states, the proof of equation (49) is more involved and only partial. We do not give the full details here as they can be found in [9] (or in section 4, where we use the same technique of proof). In a nutshell, the proof relies on a variational method similar to the procedure used in $[52,53]$. One defines the uncertainty functional

$$
F(\hat{\rho})=h(\mathbf{x})+h(\mathbf{p})-\frac{1}{2} \ln \left(\frac{\operatorname{det} \gamma_{\mathbf{x}} \operatorname{det} \gamma_{\mathbf{p}}}{\operatorname{det} \gamma}\right)
$$

and shows that any $n$-modal squeezed vacuum state is a local extremum of $F(\hat{\rho})$. Since $F(\hat{\rho})$ is invariant under $(x, p)$-displacements, it follows that all Gaussian pure states are extrema too. To complete the proof of equation (49), one must take the two following statements for granted:

(i) Pure Gaussian states are global minimizers of the uncertainty functional $F(\hat{\rho})$.

(ii) The uncertainty functional $F(\hat{\rho})$ is concave, so relation (49) is valid for mixed states.

Remark that (i) and (ii) both prevail for the uncertainty functional $h(\mathbf{x})+h(\mathbf{p})$ appearing in the entropic uncertainty relation (41).

For one mode, the entropy-power formulation of equation (50) reads

$$
\frac{N_{x} N_{p}}{\sigma_{x}^{2} \sigma_{p}^{2}} \operatorname{det} \gamma \geqslant \frac{1}{4}
$$

where $N_{x}$ and $N_{p}$ are the entropy powers defined in equation (43). This highlights the fact that the Robertson-Schrödinger relation (8) can be deduced from the tight entropy-power uncertainty relation (53). Indeed, since $N_{x} \leqslant \sigma_{x}^{2}$ and $N_{p} \leqslant \sigma_{p}^{2}$, we have the chain of inequalities 


$$
\operatorname{det} \gamma \geqslant \frac{N_{x} N_{p}}{\sigma_{x}^{2} \sigma_{p}^{2}} \operatorname{det} \gamma \geqslant \frac{1}{4}
$$

and, once again, both inequalities coincide only for Gaussian $x$ - and $p$-distributions.

For $n$ modes, the entropy-power formulation of equation (49) becomes

$$
\frac{\left(N_{\mathbf{x}} N_{\mathbf{p}}\right)^{n}}{\operatorname{det} \gamma_{\mathbf{x}} \operatorname{det} \gamma_{\mathbf{p}}} \operatorname{det} \gamma \geqslant\left(\frac{1}{4}\right)^{n}
$$

where

$$
N_{\mathbf{x}}=\frac{1}{2 \pi e} \mathrm{e}^{\frac{2}{n} h(\mathbf{x})} \quad N_{\mathbf{p}}=\frac{1}{2 \pi e} \mathrm{e}^{\frac{2}{n} h(\mathbf{p})} .
$$

Here too, we can use the fact that the maximum entropy for a fixed covariance matrix is given by the Gaussian distribution, which implies that

$$
\operatorname{det} \gamma \geqslant \frac{\left(N_{\mathbf{x}} N_{\mathbf{p}}\right)^{n}}{\operatorname{det} \gamma_{\mathbf{x}} \operatorname{det} \gamma_{\mathbf{p}}} \operatorname{det} \gamma \geqslant\left(\frac{1}{4}\right)^{n}
$$

that is, the $n$-mode tight entropy-power uncertainty relation (55) implies the $n$-mode variancebased Robertson-Schrödinger uncertainty relation, equation (13).

\subsection{Entropic uncertainty relation for arbitrary quadratures}

Traditionally, continuous-variable entropic uncertainty relations have been formulated for the position and momentum quadratures or, more precisely, for continuous variables that are related by a Fourier transform. However, as for variance-based ones, entropic uncertainty relations can be extended to any pair of variables. In 2009, Guanlei et al [54] first formulated an entropic uncertainty relation for two rotated quadratures:

\section{EUR for two rotated quadratures [54]:}

Any one-mode state $\rho$ satisfies the entropic uncertainty relation

$$
h\left(x_{\theta}\right)+h\left(x_{\phi}\right) \geqslant \ln (\pi e|\sin (\theta-\phi)|)
$$

where $\hat{x}_{\theta}=\hat{x} \cos \theta+\hat{p} \sin \theta$ and $\hat{x}_{\phi}=\hat{x} \cos \phi+\hat{p} \sin \phi$ are two rotated quadratures, and $h(\cdot)$ is the Shannon differential entropy.

In 2011, Huang [55] obtained a more general entropic uncertainty relation that holds for any pair of observables, that is, two variables that are not necessarily canonically conjugate (or that are not related by a Fourier transform):

EUR for two arbitrary quadratures [55]:

Any $n$-modal state $\rho$ satisfies the entropic uncertainty relation

$$
h(\hat{A})+h(\hat{B}) \geqslant \ln (\pi e|[\hat{A}, \hat{B}]|)
$$

where $h(\cdot)$ is the Shannon differential entropy, $\hat{A}$ and $\hat{B}$ are two observables defined as

$$
\hat{A}=\sum_{i=1}^{n}\left(a_{i} \hat{x}_{i}+a_{i}^{\prime} \hat{p}_{i}\right), \quad \hat{B}=\sum_{i=1}^{n}\left(b_{i} \hat{x}_{i}+b_{i}^{\prime} \hat{p}_{i}\right),
$$

and $[\hat{A}, \hat{B}]$ (which is a scalar) is the commutator between them. 
Obviously, if $\hat{A}=\hat{x}$ and $\hat{B}=\hat{p}$, this inequality reduces to the entropic uncertainty relation of Bialynicki-Birula and Mycielski, equation (2), while it reduces to equation (58) if $n=1$.

More recently, an entropic uncertainty relation that holds for any two vectors of not-necessarily canonically conjugated variables was derived in [56]. The bound on entropies is then expressed in terms of the determinant of a $n \times n$ matrix formed with the commutators between the $n$ measured variables:

EUR for two arbitrary vectors of intercommuting quadratures [56]:

Let $\mathbf{y}=\left(\hat{y}_{1}, \cdots \hat{y}_{n}\right)^{T}$ be a vector of commuting quadratures and $\mathbf{z}=\left(\hat{z}_{1}, \cdots \hat{z}_{n}\right)^{T}$ be another vector of commuting quadratures. Let each of the components of $\mathbf{y}$ and $\mathbf{z}$ be written as a linear combination of the $(\hat{x}, \hat{p})$ quadratures of an n-modal system, namely

$$
\begin{array}{ll}
\hat{y}_{i}=\sum_{k=1}^{n} a_{i, k} \hat{x}_{k}+\sum_{k=1}^{n} a_{i, k}^{\prime} \hat{p}_{k} & (i=1, \cdots n) \\
\hat{z}_{j}=\sum_{k=1}^{n} b_{j, k} \hat{x}_{k}+\sum_{k=1}^{n} b_{j, k}^{\prime} \hat{p}_{k} & (j=1, \cdots n) .
\end{array}
$$

Then, any n-modal state $\rho$ satisfies the entropic uncertainty relation

$$
h(\mathbf{y})+h(\mathbf{z}) \geqslant \ln \left((\pi e)^{n}|\operatorname{det} \mathbf{K}|\right)
$$

where $h(\cdot)$ stands for the Shannon differential entropy of the probability distribution of the vectors of jointly measured quadratures $\hat{y}_{i}$ 's or $\hat{z}_{j}$ 's, and $\mathbf{K}_{i j}=\left[\hat{y}_{i}, \hat{z}_{j}\right]$ denotes the $n \times n$ matrix of commutators (which are scalars).

The proof of equation (62) exploits the fact that the probability distributions of vectors $\mathbf{y}$ and $\mathbf{z}$ are related by a fractional Fourier transform (instead of a simple Fourier transform). Then, the entropic uncertainty relation of Bialynicki-Birula and Mycielski, equation (41), simply corresponds to the special case of equation (62) for a Fourier transform, that is, when measuring either all $x$ quadratures or all $p$ quadratures on $n$ modes. Also, equations (58) and (59) are special cases of equation (62) for a one-by-one matrix K. Finally, let us also mention that equation (62) still holds if we jointly measure $n$ quadratures on a larger $N$-dimensional system, i.e. when the sum over $k$ in equation (61) goes to $N$ (with $N>n$ ) [56].

By exploiting the entropy-power formulation of equation (62), it is possible to derive an $n$-dimensional extension of the usual Robertson uncertainty relation in position and momentum spaces where, instead of expressing the complementarity between observables $\hat{A}$ and $\hat{B}$ (which are linear combinations of quadratures, so the commutator $[\hat{A}, \hat{B}]$ is a scalar), one expresses the complementarity between two vectors of intercommuting observables. Defining the entropy powers of $\mathbf{y}$ and $\mathbf{z}$ as

$$
N_{\mathbf{y}}=\frac{1}{2 \pi e} \mathrm{e}^{\frac{2}{n} h(\mathbf{y})}, \quad N_{\mathbf{z}}=\frac{1}{2 \pi e} \mathrm{e}^{\frac{2}{n} h(\mathbf{z})},
$$

we can rewrite equation (62) as an entropy-power uncertainty relation for two arbitrary vectors of intercommuting quadratures $\mathbf{y}$ and $\mathbf{z}$, namely

$$
N_{\mathbf{y}} N_{\mathbf{z}} \geqslant \frac{|\operatorname{det} \mathbf{K}|^{2 / n}}{4} \text {. }
$$

Again, we may use the fact that the maximum entropy for a fixed covariance matrix is reached by the Gaussian distribution and write $N_{\mathbf{y}} \leqslant\left(\operatorname{det} \boldsymbol{\Gamma}_{\mathbf{y}}\right)^{1 / n}$ and $N_{\mathbf{z}} \leqslant\left(\operatorname{det} \boldsymbol{\Gamma}_{\mathbf{z}}\right)^{1 / n}$, where 
$\left(\boldsymbol{\Gamma}_{\mathbf{y}}\right)_{i j}=\left\langle\left\{\hat{y}_{i}, \hat{y}_{j}\right\}\right\rangle / 2-\left\langle\hat{y}_{i}\right\rangle\left\langle\hat{y}_{j}\right\rangle$ and $\left(\boldsymbol{\Gamma}_{\mathbf{z}}\right)_{i j}=\left\langle\left\{\hat{z}_{i}, \hat{z}_{j}\right\}\right\rangle / 2-\left\langle\hat{z}_{i}\right\rangle\left\langle\hat{z}_{j}\right\rangle$ are the (reduced) covariance matrices of the $\hat{y}_{i}$ and $\hat{z}_{i}$ quadratures. Combining these inequalities with equation (64), we obtain the $n$-modal variance-based uncertainty relation (VUR):

VUR for two arbitrary vectors of intercommuting quadratures [56]:

Let $\mathbf{y}=\left(\hat{y}_{1}, \cdots \hat{y}_{n}\right)^{T}$ be a vector of commuting quadratures, $\mathbf{z}=\left(\hat{z}_{1}, \cdots \hat{z}_{n}\right)^{T}$ be another vector of commuting quadratures, and let each of the components of these vectors be written as a linear combination of the $(\hat{x}, \hat{p})$ quadratures of an $N$-modal system $(N \geqslant n)$. Then, any $N$-modal state $\rho$ verifies the variance-based uncertainty relation

$$
\operatorname{det} \boldsymbol{\Gamma}_{\mathbf{y}} \operatorname{det} \boldsymbol{\Gamma}_{\mathbf{z}} \geqslant \frac{|\operatorname{det} \mathbf{K}|^{2}}{4^{n}}
$$

where $\boldsymbol{\Gamma}_{\mathbf{y}}\left(\boldsymbol{\Gamma}_{\mathbf{z}}\right)$ is the covariance matrix of the jointly measured quadratures $\hat{y}_{i}{ }^{\prime} s\left(\hat{z}_{j}{ }^{\prime} s\right)$, and $\mathbf{K}_{i j}=\left[\hat{y}_{i}, \hat{z}_{j}\right]$ denotes the $n \times n$ matrix of commutators (which are scalars).

Just like equation (41) can be extended to equation (47), the entropic uncertainty relation (62) can also be extended to Rényi entropies:

Rényi EUR for two arbitrary vectors of intercommuting quadratures [56]:

Let $\mathbf{y}=\left(\hat{y}_{1}, \cdots \hat{y}_{n}\right)^{T}$ be a vector of commuting quadratures, $\mathbf{z}=\left(\hat{z}_{1}, \cdots \hat{z}_{n}\right)^{T}$ be another vector of commuting quadratures, and let each of the components of these vectors be written as a linear combination of the $(\hat{x}, \hat{p})$ quadratures of an $N$-modal system $(N \geqslant n)$. Then, any $N$-modal state $\rho$ verifies the Rényi entropic uncertainty relation

$$
h_{\alpha}(\mathbf{y})+h_{\beta}(\mathbf{z}) \geqslant n \ln (\pi)+\frac{n \ln (\alpha)}{2(\alpha-1)}+\frac{n \ln (\beta)}{2(\beta-1)}+\ln |\operatorname{det} \mathbf{K}|
$$

with

$$
\frac{1}{\alpha}+\frac{1}{\beta}=2, \quad \alpha>0, \quad \beta>0
$$

where $h_{\alpha}(\cdot)$ stands for the Rényi entropy of the probability distributions of the vectors of jointly measured quadratures $\hat{y}_{i}$ 's or $\hat{z}_{j}$ 's, and $\mathbf{K}_{i j}=\left[\hat{y}_{i}, \hat{z}_{j}\right]$ is the $n \times n$ matrix of commutators (which are scalars).

As expected, in the limit where $\alpha \rightarrow 1$ and $\beta \rightarrow 1$, we recover the uncertainty relations for Shannon differential entropies, equation (62). Moreover, in the one-dimensional case ( $N=n=1$ ), equation (66) coincides with the result found in [57].

\subsection{Other entropic uncertainty relations}

Let us conclude this section by mentioning a few related entropic uncertainty relations. First, it is also possible to express with entropies the complementary between the pair of variables $\left(\phi, L_{z}\right)$, that is, a (continuous) angle and associated (discrete) angular momentum [58], or $(\phi, \hat{N})$, that is, a (continuous) phase and associated (discrete) number operator [53, 59]. Unlike those considered in the present paper, such entropic uncertainty relations for $\left(\phi, L_{z}\right)$ or $(\phi, \hat{N})$ may be viewed as hybrid as they mix discrete and continuous entropies. Similarly, Hall considered an entropic time-energy uncertainty relation for bound quantum systems (thus having discrete energy eigenvalues), which expresses the balance between a discrete entropy for the energy distribution and a continuous entropy for the time shift applied to the system [60]. 
Recently, an entropic time-energy uncertainty relation has also been formulated for general time-independent Hamiltonians, where the time uncertainty is associated with measuring the (continuous) time state of a quantum clock [61].

As already mentioned, another variant of entropic uncertainty relations can be defined in the presence of quantum memory. This situation, where the observer may exploit some side information, has been analyzed in the case of position and momentum variables by Furrer et al [62]. Still another interesting scenario concerns uncertainties occurring in successive measurements. While the most common uncertainty relations assume that one repeats measurements on the same state (as we do throughout this paper), one may consider successive measurements on a system whose state evolves as a result of the measurements. The entropic uncertainty relations for canonically conjugate variables in this scenario have been derived by Rastegin [63].

This closes our review on continuous-variable entropic uncertainty relations. In the rest of this paper, we present some new results, namely a tight entropic uncertainty relation for two vectors of quadratures (section 4) and several conjectures for new entropic uncertainty relations involving more than two variables (section 5).

\section{Tight entropic uncertainty relation for arbitrary quadratures}

\subsection{Minimum uncertainty states}

We now build an entropic uncertainty relation which holds for any two vectors of intercommuting quadratures and is saturated by all pure Gaussian states (hence, we call it tight). It combines the two previous results, namely equations (49) and (62).

Let us stress that equation (62) is not saturated by pure Gaussian states, in general, so the idea here is to take correlations into account following a similar procedure as the one leading to equation (49). One can easily understand the problem by considering the one mode case, equation (58), which can also be written as

$$
h(x)+h\left(x_{\theta}\right) \geqslant \ln (\pi e|\sin \theta|) .
$$

We compute $h(x)+h\left(x_{\theta}\right)$ for a general pure Gaussian state (i.e. a squeezed state with parameter $r$ and angle $\phi$ ) with covariance matrix

$$
\gamma=\frac{1}{2}\left(\begin{array}{ll}
\mathrm{e}^{-2 r} \cos ^{2} \phi+\mathrm{e}^{2 r} \sin ^{2} \phi & \left(\mathrm{e}^{2 r}-\mathrm{e}^{-2 r}\right) \cos \phi \sin \phi \\
\left(\mathrm{e}^{2 r}-\mathrm{e}^{-2 r}\right) \cos \phi \sin \phi & \mathrm{e}^{2 r} \cos ^{2} \phi+\mathrm{e}^{-2 r} \sin ^{2} \phi
\end{array}\right)
$$

and plot it as a function of $\phi$ (we fix $r=0.2$ and consider several values of $\theta$ ). As we can see on figure 2 , where the solid lines represent the sum of entropies (for $\theta=\pi / 4, \pi / 2$ and $5 \pi / 3$ ) and the dashed lines stand for the corresponding lower bound $\ln (\pi e|\sin \theta|)$, the uncertainty relation (68) is in general not saturated by any pure Gaussian state. The only exception is $\theta=\pi / 2$, namely the Bialynicki-Birula and Mycielski uncertainty relation (2), which is only saturated when the state is aligned with the principal axes, i.e. if $\phi=0, \pi / 2, \pi$ or $3 \pi / 2$.

This suggests that a modification of equation (62) is needed in order to impose that the Gaussian pure states become minimum-uncertainty states, as they are in equation (49).

\subsection{Entropic uncertainty relation saturated by all pure Gaussian states}

Let $\mathbf{y}=\left(\hat{y}_{1}, \cdots \hat{y}_{n}\right)^{T}$ be a vector of commuting quadratures and $\mathbf{z}=\left(\hat{z}_{1}, \cdots \hat{z}_{n}\right)^{T}$ be another vector of commuting quadratures. Let us suppose that they correspond to the output $x$-quadratures obtained after applying two possible symplectic transformations onto some $n$-modal 


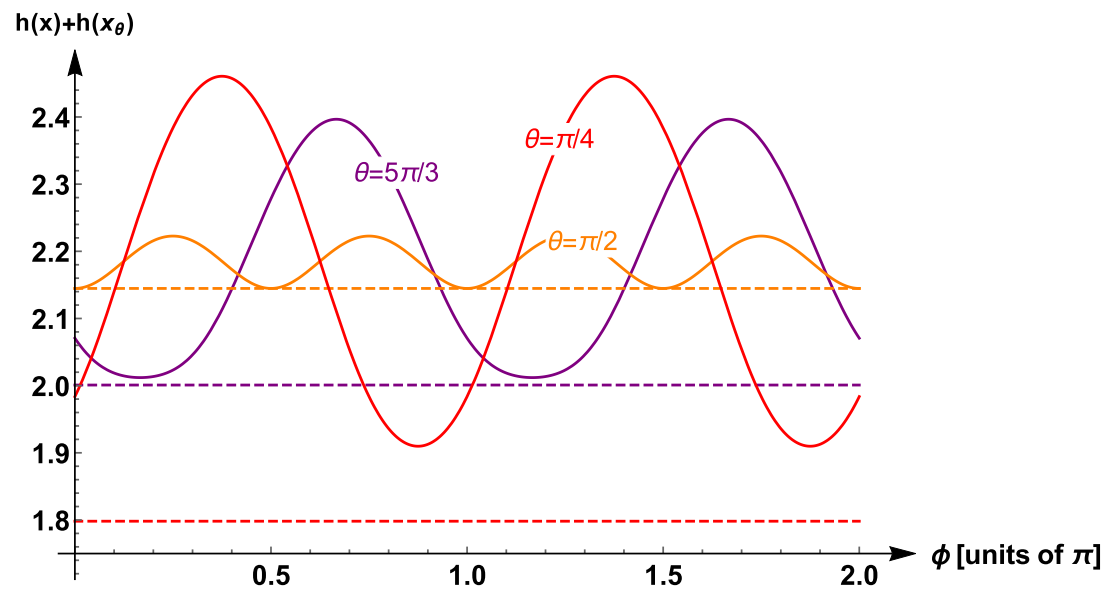

Figure 2. Plot of equation (68) for pure Gaussian states, illustrating that this entropic uncertainty relation is, in general, not saturated. Solid lines represent the sum of entropies for $\theta=\pi / 4, \pi / 2$ and $5 \pi / 3$, while dashed lines show the corresponding lower bounds $\ln (\pi e|\sin \theta|)$.

system. In other words, the $2 n$-dimensional vector of input quadratures $\left(\hat{x}_{1}, \cdots, \hat{x}_{n}, \hat{p}_{1}, \cdots, \hat{p}_{n}\right)^{T}$ is transformed into the $2 n$-dimensional vector of output quadratures $\left(\hat{y}_{1}, \cdots, \hat{y}_{n}, \hat{q}_{1}, \cdots, \hat{q}_{n}\right)^{T}$ or $\left(\hat{z}_{1}, \cdots, \hat{z}_{n}, \hat{o}_{1}, \cdots, \hat{o}_{n}\right)^{T}$, where $\left(\hat{q}_{1}, \cdots, \hat{q}_{n}\right)^{T}$ (resp. $\left.\left(\hat{o}_{1}, \cdots, \hat{o}_{n}\right)^{T}\right)$ is a vector of quadratures that are pairwise canonically conjugate with $\left(\hat{y}_{1}, \cdots, \hat{y}_{n}\right)^{T}\left(\operatorname{resp} .\left(\hat{z}_{1}, \cdots, \hat{z}_{n}\right)^{T}\right)$. As for the $x, p$ quadratures, it is possible to define a covariance matrix $\Gamma$ for the $y, z$ quadratures. Its elements are expressed as

$$
\Gamma_{i j}=\frac{1}{2}\left\langle\hat{R}_{i} \hat{R}_{j}+\hat{R}_{j} \hat{R}_{i}\right\rangle-\left\langle\hat{R}_{i}\right\rangle\left\langle\hat{R}_{j}\right\rangle
$$

with $\mathbf{R}=\left(\hat{y}_{1}, \ldots, \hat{y}_{n}, \hat{z}_{1}, \ldots, \hat{z}_{n}\right)^{T}$. The knowledge of $\boldsymbol{\Gamma}$ allows us to take correlations into account and write a general form of the entropic uncertainty relation for any two vectors of intercommuting quadratures:

Tight EUR for two arbitrary vectors of intercommuting quadrature:

Let $\mathbf{y}=\left(\hat{y}_{1}, \cdots \hat{y}_{n}\right)^{T}$ be a vector of commuting quadratures, $\mathbf{z}=\left(\hat{z}_{1}, \cdots \hat{z}_{n}\right)^{T}$ be another vector of commuting quadratures, and let each of the components of $\mathbf{y}$ and $\mathbf{z}$ be written as a linear combination of the $(\hat{x}, \hat{p})$ quadratures of an $n$-modal system. Then, any $n$-modal state $\rho$ satisfies the entropic uncertainty relation ${ }^{8}$

$$
h(\mathbf{y})+h(\mathbf{z})-\frac{1}{2} \ln \left(\frac{\operatorname{det} \boldsymbol{\Gamma}_{\mathbf{y}} \operatorname{det} \boldsymbol{\Gamma}_{\mathbf{z}}}{\operatorname{det} \boldsymbol{\Gamma}}\right) \geqslant \ln \left((\pi e)^{n}|\operatorname{det} \mathbf{K}|\right)
$$

where $h(\cdot)$ stands for the Shannon differential entropy of the probability distribution of the vector of jointly measured quadratures $\hat{y}_{i}$ 's or $\hat{z}_{j}$ 's, $\Gamma$ is the covariance matrix defined in equation (70), $\boldsymbol{\Gamma}_{\mathbf{y}}$ and $\boldsymbol{\Gamma}_{\mathbf{z}}$ are the reduced covariance matrices of the $\hat{y}_{i}$ and $\hat{z}_{i}$ quadratures, respectively, and $\mathbf{K}_{i j}=\left[\hat{y}_{i}, \hat{z}_{j}\right]$ is the $n \times n$ matrix of commutators (which are scalars). The saturation is obtained when $\rho$ is Gaussian and pure.

\footnotetext{
${ }^{8}$ The proof is conditional on two reasonable assumptions, see below.
} 
Let us first remark that equation (71) is invariant under displacements. Indeed, the differential entropy is invariant under displacements (see equation (30)), and so are $\boldsymbol{\Gamma}, \boldsymbol{\Gamma}_{\mathbf{y}}$ and $\boldsymbol{\Gamma}_{\mathbf{z}}$ as is obvious from their definitions. Thus, in the proof of equation (71), we can restrict to states centered at the origin. As we will see in section 4.4, our proof is based on a variational method used to show that pure Gaussian states extremize the uncertainty functional

$$
F(\hat{\rho})=h(\mathbf{y})+h(\mathbf{z})-\frac{1}{2} \ln \left(\frac{\operatorname{det} \boldsymbol{\Gamma}_{\mathbf{y}} \operatorname{det} \boldsymbol{\Gamma}_{\mathbf{z}}}{\operatorname{det} \boldsymbol{\Gamma}}\right) .
$$

The proof, however, is partial as it relies on two assumptions:

(i) Pure Gaussian states are global minimizers of the uncertainty functional $F(\hat{\rho})$.

(ii) The uncertainty functional $F(\hat{\rho})$ is concave, so relation (71) is valid for mixed states.

\subsection{Special case of Gaussian states}

Before addressing the proof of equation (71) with a variational method, let us see how this entropic uncertainty relation applies to Gaussian states. In particular, let us prove first that equation (71) is saturated by all pure Gaussian states. Then, we will show that for all Gaussian states, it can be proven using the $n$-modal version of the Robertson-Schrödinger uncertainty relation, equation (13).

Consider a pure $n$-modal Gaussian state. Its Wigner function is given by

$$
W^{\mathrm{G}}(x, p)=\frac{1}{\pi^{n}} \mathrm{e}^{-\frac{1}{2} \mathbf{r}^{T} \gamma^{-1} \mathbf{r}}
$$

and its covariance matrix is expressed as

$$
\gamma=\left(\begin{array}{ll}
\gamma_{\mathbf{x}} & \gamma_{\mathbf{x p}} \\
\gamma_{\mathbf{x p}} & \gamma_{\mathbf{p}}
\end{array}\right)_{2 n \times 2 n}
$$

where $\gamma_{\mathbf{x}}$ and $\gamma_{\mathbf{p}}$ are the reduced covariance matrices of the position and momentum quadratures. Since the state is pure and Gaussian, det $\gamma=(1 / 4)^{n}$.

To evaluate equation (71) we need to find the determinant of the covariance matrix $\Gamma$ for the $y, z$-quadratures. The calculation is reported in appendix B and leads to

$$
\operatorname{det} \boldsymbol{\Gamma}=\operatorname{det} \gamma|\operatorname{det} \mathbf{K}|^{2} \text {. }
$$

Note that equation (75) is true for any state, Gaussian or not, but since we are dealing with a pure Gaussian state, $\operatorname{det} \gamma=1 / 4^{n}$, it simplifies to

$$
\operatorname{det} \boldsymbol{\Gamma}=\frac{1}{4^{n}}|\operatorname{det} \mathbf{K}|^{2} \text {. }
$$

The last step needed to evaluate equation (71) is to compute the differential entropies of the $y$ and $z$ quadratures. Since these quadratures are obtained after applying some symplectic transformations, the Wigner function, which is Gaussian for the input state, remains Gaussian for the output state. The probability distributions of the jointly measured quadratures $\hat{y}_{i}$ or $\hat{z}_{j}$ are thus given by the following Gaussian distributions

$$
P(\mathbf{y})=\frac{1}{\sqrt{(2 \pi)^{n} \operatorname{det} \boldsymbol{\Gamma}_{\mathbf{y}}}} \mathrm{e}^{-\frac{1}{2} \mathbf{y}^{T} \boldsymbol{\Gamma}_{\mathbf{y}}^{-1} \mathbf{y}}, \quad P(\mathbf{z})=\frac{1}{\sqrt{(2 \pi)^{n} \operatorname{det} \boldsymbol{\Gamma}_{\mathbf{z}}}} \mathrm{e}^{-\frac{1}{2} \mathbf{z}^{T} \boldsymbol{\Gamma}_{\mathbf{z}}^{-1} \mathbf{z}}
$$

and we easily evaluate the corresponding differential entropies 


$$
h(\mathbf{y})=\frac{1}{2} \ln \left((2 \pi e)^{n} \operatorname{det} \boldsymbol{\Gamma}_{\mathbf{y}}\right), \quad h(\mathbf{z})=\frac{1}{2} \ln \left((2 \pi e)^{n} \operatorname{det} \boldsymbol{\Gamma}_{\mathbf{z}}\right) .
$$

Inserting these quantities together with equation (76) into the left-hand side of equation (71) yields the lower bound $\ln \left((\pi e)^{n}|\operatorname{det} \mathbf{K}|\right)$, so we have proved that Gaussian pure states are minimum uncertainty states of equation (71), as desired.

Let us emphasize that the entropic uncertainty relation that does not take correlations into account, equation (62), is only saturated by pure Gaussian states with vanishing correlations. Indeed, for pure Gaussian states, we find that

$$
h(\mathbf{y})+h(\mathbf{z})=\ln \left((2 \pi e)^{n} \sqrt{\operatorname{det} \boldsymbol{\Gamma}_{\mathbf{y}} \operatorname{det} \boldsymbol{\Gamma}_{\mathbf{z}}}\right)
$$

reaches the lower bound of equation (62) only if

$$
\begin{array}{rlrl} 
& & 2^{n} \sqrt{\operatorname{det} \boldsymbol{\Gamma}_{\mathbf{y}} \operatorname{det} \boldsymbol{\Gamma}_{\mathbf{z}}} & =|\operatorname{det} \mathbf{K}| \\
\Leftrightarrow & 2^{n} \sqrt{\operatorname{det} \boldsymbol{\Gamma}_{\mathbf{y}} \operatorname{det} \boldsymbol{\Gamma}_{\mathbf{z}}} & =2^{n} \sqrt{\operatorname{det} \boldsymbol{\Gamma}} \\
\Leftrightarrow & & \operatorname{det} \boldsymbol{\Gamma}_{\mathbf{y}} \operatorname{det} \boldsymbol{\Gamma}_{\mathbf{z}} & =\operatorname{det} \boldsymbol{\Gamma}
\end{array}
$$

where we have used equation (76). Obviously, this is only true when $\boldsymbol{\Gamma}_{y z}=0$, i.e. when there is no correlation between the $y_{i}$ and $z_{i}$ quadratures. This confirms that the entropic uncertainty relation (62) is not saturated by all pure Gaussian states, as we had explicitly checked for one mode in figure 2.

Second, let us now prove that equation (71) holds for a general mixed Gaussian state. For any Gaussian state, pure or not, the differential entropies are still given by equation (78), so that

$$
h(\mathbf{y})+h(\mathbf{z})-\frac{1}{2}\left(\frac{\operatorname{det} \boldsymbol{\Gamma}_{\mathbf{y}} \operatorname{det} \boldsymbol{\Gamma}_{\mathbf{z}}}{\operatorname{det} \boldsymbol{\Gamma}}\right)=\ln \left((2 \pi e)^{n} \sqrt{\operatorname{det} \boldsymbol{\Gamma}}\right) .
$$

Using equation (75) together with the $n$-modal version of the Robertson-Schrödinger uncertainty relation, $\operatorname{det} \gamma \geqslant 1 / 4^{n}$, we get

$$
\sqrt{\operatorname{det} \boldsymbol{\Gamma}} \geqslant \frac{|\operatorname{det} \mathbf{K}|}{2^{n}}
$$

Injecting this inequality into equation (81) complete the proof of equation (71) for all Gaussian states.

\subsection{Partial proof for all states}

The difficult part is to verify the entropic uncertainty relation for a general-not necessarily Gaussian - state. Inspired from [9], we give here a partial proof of equation (71) based on a variational method (see $[52,53]$ ), which is conditional on two assumptions (see assumptions (i) and (ii) in section 4.2). More precisely, we seek a pure state $\hat{\rho}=|\psi\rangle\langle\psi|$ that extremizes our uncertainty functional (72) and show that any pure Gaussian state is such an extremum. The steps of the proof are similar to those developed in [9], except that we consider the $y, z$-quadratures instead of the $x, p$-quadratures. The assumptions are also the same.

As already mentioned, $F(|\psi\rangle)$ is invariant under displacements so that we can restrict our search to extremal states centered on 0 . We also require extremal state to be normalized. Accounting for these constraints by using the Lagrange multipliers method, we have to solve $\frac{\partial J}{\partial\langle\psi|}=0$ with 
$J=h(\mathbf{y})+h(\mathbf{z})-\frac{1}{2} \ln \left(\frac{\operatorname{det} \boldsymbol{\Gamma}_{\mathbf{y}} \operatorname{det} \boldsymbol{\Gamma}_{\mathbf{z}}}{\operatorname{det} \boldsymbol{\Gamma}}\right)+\lambda(\langle\psi \mid \psi\rangle-1)+\sum_{i=1}^{2 n} \mu_{i}\left\langle\psi\left|\hat{R}_{i}\right| \psi\right\rangle$,

where $\lambda$ and $\mu_{i}$ are Lagrange multipliers. Note that, as explained in [9], it is not necessary to consider $\frac{\partial J}{\partial|\psi\rangle}=0$ since no additional information would be obtained.

Let us evaluate the derivative of each term of equation (83) separately. First, the derivative of $h(\mathbf{y})$ gives

$$
\begin{aligned}
\frac{\partial h(\mathbf{y})}{\partial\langle\psi|} & =\frac{\partial}{\partial\langle\psi|}\left(\int P(\mathbf{y}) \ln P(\mathbf{y}) \mathrm{d} \mathbf{y}\right) \\
& =\frac{\partial}{\partial\langle\psi|}\left(\int\langle\psi \mid \mathbf{y}\rangle\langle\mathbf{y} \mid \psi\rangle \ln (\langle\psi \mid \mathbf{y}\rangle\langle\mathbf{y} \mid \psi\rangle) \mathrm{d} \mathbf{y}\right) \\
& =-(\ln P(\mathbf{y})+1)|\psi\rangle
\end{aligned}
$$

and similarly for $h(\mathbf{z})$. Note that $\mathbf{y}$ in the last line of equation (84) denotes a vector of quadrature operators, so that $\ln P(\mathbf{y})$ is an operator too. With the help of Jacobi's formula [64], the derivatives of the determinant of the three covariance matrices give

$$
\begin{aligned}
\frac{\partial}{\partial\langle\psi|} \ln \operatorname{det} \boldsymbol{\Gamma}_{\mathbf{y}} & =\frac{1}{\operatorname{det} \boldsymbol{\Gamma}_{\mathbf{y}}} \frac{\partial}{\partial\langle\psi|} \operatorname{det} \boldsymbol{\Gamma}_{\mathbf{y}} \\
& =\frac{1}{\operatorname{det} \boldsymbol{\Gamma}_{\mathbf{y}}} \operatorname{Tr}\left[\left(\operatorname{det} \boldsymbol{\Gamma}_{\mathbf{y}}\right) \boldsymbol{\Gamma}_{\mathbf{y}}^{-1} \frac{\partial \boldsymbol{\Gamma}_{\mathbf{y}}}{\partial\langle\psi|}\right] \\
& =\sum_{i=1}^{n} \sum_{k=1}^{n}\left(\boldsymbol{\Gamma}_{\mathbf{y}}\right)_{i k}^{-1} \frac{\partial\left(\boldsymbol{\Gamma}_{\mathbf{y}}\right)_{k i}}{\partial\langle\psi|} \\
& =\sum_{i=1}^{n} \sum_{k=1}^{n}\left(\boldsymbol{\Gamma}_{\mathbf{y}}\right)_{i k}^{-1} \frac{\left(\hat{y}_{k} \hat{y}_{i}+\hat{y}_{i} \hat{y}_{k}\right)}{2}|\psi\rangle \\
& =\left[\sum_{i=1}^{n} \sum_{k=1}^{n} \frac{\hat{y}_{k}\left(\boldsymbol{\Gamma}_{\mathbf{y}}\right)_{i k}^{-1} \hat{y}_{i}}{2}+\sum_{i=1}^{n} \sum_{k=1}^{n} \frac{\hat{y}_{i}\left(\boldsymbol{\Gamma}_{\mathbf{y}}\right)_{i k}^{-1} \hat{y}_{k}}{2}\right]|\psi\rangle \\
& =\mathbf{y}^{T} \boldsymbol{\Gamma}_{\mathbf{y}}^{-1} \mathbf{y}|\psi\rangle
\end{aligned}
$$

and similarly

$$
\begin{aligned}
\frac{\partial}{\partial\langle\psi|} \ln \operatorname{det} \boldsymbol{\Gamma}_{\mathbf{z}} & =\mathbf{z}^{T} \boldsymbol{\Gamma}_{\mathbf{z}}^{-1} \mathbf{z}|\psi\rangle \\
\frac{\partial}{\partial\langle\psi|} \ln \operatorname{det} \boldsymbol{\Gamma} & =\mathbf{R}^{T} \boldsymbol{\Gamma}^{-1} \mathbf{R}|\psi\rangle .
\end{aligned}
$$

Finally, the derivatives of the last two terms of equation (83) give

$$
\frac{\partial}{\partial\langle\psi|}\left(\lambda(\langle\psi \mid \psi\rangle-1)+\sum_{i=1}^{2 n} \mu_{i}\left\langle\psi\left|\hat{R}_{i}\right| \psi\right\rangle\right)=\left(\lambda+\sum_{i=1}^{2 n} \mu_{i} \hat{R}_{i}\right)|\psi\rangle
$$

so that the variational equation can be rewritten as an eigenvalue equation for $|\psi\rangle$,

$$
\begin{aligned}
{[-\ln P(\mathbf{y})-\ln P(\mathbf{z})-2+\lambda} & +\sum_{i=1}^{2 n} \mu_{i} \hat{R}_{i}-\frac{1}{2} \mathbf{y}^{T} \boldsymbol{\Gamma}_{\mathbf{y}}^{-1} \mathbf{y} \\
& \left.-\frac{1}{2} \mathbf{z}^{T} \boldsymbol{\Gamma}_{\mathbf{z}}^{-1} \mathbf{y}+\frac{1}{2} \mathbf{R}^{T} \boldsymbol{\Gamma}^{-1} \mathbf{R}\right]|\psi\rangle=0 .
\end{aligned}
$$


Thus, the states $|\psi\rangle$ extremizing $F(|\psi\rangle)$ are the eigenstates of equation (88).

Now, instead of looking for all eigenstates, we show that all pure Gaussian states are solution of equation (88). We have already written the probability distributions $P(\mathbf{y})$ and $P(\mathbf{z})$ for a $n$-modal pure Gaussian state (see equation (77)), so we have

$$
\ln P(\mathbf{y})+\ln P(\mathbf{z})=-\ln \left((2 \pi)^{n} \sqrt{\operatorname{det} \boldsymbol{\Gamma}_{\mathbf{y}} \operatorname{det} \boldsymbol{\Gamma}_{\mathbf{z}}}\right)-\frac{1}{2} \mathbf{y}^{T} \boldsymbol{\Gamma}_{\mathbf{y}}^{-1} \mathbf{y}-\frac{1}{2} \mathbf{z}^{T} \boldsymbol{\Gamma}_{\mathbf{z}}^{-1} \mathbf{z}
$$

and the eigenvalue equation (88) reduces to

$$
\left[\ln \left((2 \pi)^{n} \sqrt{\operatorname{det} \boldsymbol{\Gamma}_{\mathbf{y}} \operatorname{det} \boldsymbol{\Gamma}_{\mathbf{z}}}\right)-2+\lambda+\sum_{i=1}^{2 n} \mu_{i} \hat{R}_{i}+\frac{1}{2} \mathbf{R}^{T} \boldsymbol{\Gamma}^{-1} \mathbf{R}\right]|\psi\rangle=0 .
$$

As shown in appendix C, pure $n$-modal Gaussian states (centered on the origin) are eigenvectors of $\frac{1}{2} \mathbf{R}^{T} \boldsymbol{\Gamma}^{-1} \mathbf{R}$ with eigenvalue $n$, that is

$$
\frac{1}{2} \mathbf{R}^{T} \boldsymbol{\Gamma}^{-1} \mathbf{R}|\psi\rangle=n|\psi\rangle,
$$

so that equation (90) can be further simplified to

$$
\left[\ln \left((2 \pi)^{n} \sqrt{\operatorname{det} \boldsymbol{\Gamma}_{\mathbf{y}} \operatorname{det} \boldsymbol{\Gamma}_{\mathbf{z}}}\right)+n-2+\lambda+\sum_{i=1}^{2 n} \mu_{i} \hat{R}_{i}\right]|\psi\rangle=0 .
$$

The value of $\lambda$ is found by multiplying this equation on the left by $\langle\psi|$ and using the normalization constraint $\langle\psi \mid \psi\rangle=1$, as well as the fact that the mean values vanish, $\left\langle\psi\left|\hat{R}_{i}\right| \psi\right\rangle=0$ for all $i$, so that we are left with

$$
\left[\sum_{i=1}^{2 n} \mu_{i} \hat{R}_{i}\right]|\psi\rangle=0
$$

which is satisfied if we set all the $\mu_{i}=0$.

In summary, we have proved that there exists an appropriate choice for $\lambda$ and $\mu_{i}$ such that any pure Gaussian state centered on the origin is an extremum of the uncertainty functional $F(|\psi\rangle$ ), that is, any $n$-modal squeezed vacuum state (with arbitrary squeezing and orientation) extremizes $F(|\psi\rangle)$. Since this functional is invariant under displacement, this feature extends to all pure Gaussian states. According to Assumption (i), we take for granted that pure Gaussian states are not just local extrema, but global minima of the uncertainty functional. The last step is simply to evaluate the functional for Gaussian pure states and see that it yields $\ln \left((\pi e)^{n}|\operatorname{det} \mathbf{K}|\right)$, as shown in section 4.3. This completes the proof of equation (71) for pure states. To complete the proof for mixed states, we resort to Assumption (ii): if the functional $F(\hat{\rho})$ is concave and equation (71) holds for pure state, then it is necessarily true for mixed states too.

\subsection{Alternative formulation}

Interestingly, using the relation between $\operatorname{det} \boldsymbol{\Gamma}$ and $\operatorname{det} \mathbf{K}$ exhibited by equation (75), we can rewrite our tight entropic uncertainty relation (71) without the explicit dependence on the commutator matrix $\mathbf{K}$, that is 


$$
h(\mathbf{y})+h(\mathbf{z})-\frac{1}{2} \ln \left(\frac{\operatorname{det} \boldsymbol{\Gamma}_{\mathbf{y}} \operatorname{det} \boldsymbol{\Gamma}_{\mathbf{z}}}{\operatorname{det} \gamma}\right) \geqslant \ln \left((\pi e)^{n}\right) .
$$

Here $\gamma$ is the covariance matrix for the $x, p$-quadratures, while $\boldsymbol{\Gamma}_{\mathbf{y}}$ and $\boldsymbol{\Gamma}_{\mathbf{z}}$ are the reduced covariance matrices of the $y, z$ quadratures. If we know $\gamma$ and the symplectic transformations leading to $\mathbf{y}$ and $\mathbf{z}$, it is straightforward to access $\boldsymbol{\Gamma}_{\mathbf{y}}$ and $\boldsymbol{\Gamma}_{\mathbf{z}}$ through equation (B.8), which makes the computation of equation (94) easier. Note also that this alternative formulation becomes very similar to the tight entropic uncertainty relation for canonically-conjugate variables $\mathbf{x}$ and $\mathbf{p}$, equation (49), where we simply substitute $\boldsymbol{\Gamma}_{\mathbf{y}}$ for $\gamma_{\mathbf{x}}$ and $\boldsymbol{\Gamma}_{\mathbf{z}}$ for $\gamma_{\mathbf{p}}$.

\subsection{Entropy-power formulation and covariance-based uncertainty relation}

Following the same procedure as before, we may exploit the entropy-power formulation in order to rewrite equation (71) as

$$
N_{\mathbf{y}} N_{\mathbf{z}}\left(\frac{\operatorname{det} \boldsymbol{\Gamma}}{\operatorname{det} \boldsymbol{\Gamma}_{\mathbf{y}} \operatorname{det} \boldsymbol{\Gamma}_{\mathbf{z}}}\right)^{1 / n} \geqslant \frac{|\operatorname{det} \mathbf{K}|^{2 / n}}{4}
$$

which is a tight entropy-power uncertainty relation for two arbitrary vectors of intercommuting quadratures $\mathbf{y}$ and $\mathbf{z}$. This entropy-power formulation helps us better see that the tight entropic uncertainty relation equation (71) implies equation (62). Indeed, since $\operatorname{det} \boldsymbol{\Gamma}_{\mathbf{y}} \operatorname{det} \boldsymbol{\Gamma}_{\mathbf{z}} \geqslant \operatorname{det} \boldsymbol{\Gamma},{ }^{9}$ we see that equation (95) corresponds to lifting up the lower bound on $N_{\mathbf{y}} N_{\mathbf{z}}$ in equation (64) by a term that accounts for the $\mathbf{y}, \mathbf{z}$ correlations. Thus equation (95) implies equation (64), which is the entropy-power version of equation (62).

Now, we again use the fact that the maximum entropy for a fixed covariance matrix is reached by the Gaussian distribution, so that we can upper bound $N_{\mathbf{y}} N_{\mathbf{z}}$ by $\left(\operatorname{det} \boldsymbol{\Gamma}_{\mathbf{y}} \operatorname{det} \boldsymbol{\Gamma}_{\mathbf{z}}\right)^{1 / n}$. Combining this with equation (95), we obtain the variance-based uncertainty relation for two arbitrary vectors of quadratures $\mathbf{y}$ and $\mathbf{z}$,

$$
\operatorname{det} \boldsymbol{\Gamma} \geqslant \frac{|\operatorname{det} \mathbf{K}|^{2}}{4^{n}}
$$

which generalizes equation (65) as it takes the $\mathbf{y}, \mathbf{z}$ correlations into account.

Interestingly, equation (96) is nothing else but a special case of the Robertson uncertainty relation (14). Indeed, we see that the definition of $\boldsymbol{\Gamma}$ in equation (70) coincides with that of equation (15), with $m=2 n$. Here, we have $R_{i}=y_{i}$ and $R_{n+i}=z_{i}$ for $i=1, \cdots, n$, so that the matrix $\mathbf{C}$ defined in equation (15) can be written in terms of $\mathbf{K}_{i j}=\left[\hat{y}_{i}, \hat{z}_{j}\right]$ as

$$
\mathbf{C}=-\frac{\mathrm{i}}{2}\left(\begin{array}{cc}
0_{n \times n} & \mathbf{K} \\
-\mathbf{K} & 0_{n \times n}
\end{array}\right) \text {. }
$$

Therefore,

$$
\operatorname{det} \mathbf{C}=\left(-\frac{\mathrm{i}}{2}\right)^{2 n}(\operatorname{det} \mathbf{K})^{2}=\frac{|\operatorname{det} \mathbf{K}|^{2}}{4^{n}}
$$

where we used the fact that the $\mathbf{K}_{i j}$ 's are all pure imaginary numbers, implying that equation (14) reduces to equation (96) in this case.

\footnotetext{
${ }^{9}$ This is a generalization of Hadamard's inequality, equation (37).
} 


\section{Entropic uncertainty relations for more than two observables}

All entropic uncertainty relations considered in sections 3 and 4 address the case of two variables (or two vectors consisting each of $n$ commuting variables). Here, we turn to entropic uncertainty relations for more than two variables. As already mentioned, the entropy-power formulation is convenient to show that, in general, an entropic uncertainty relation implies a variance-based one. In particular, we showed in section 4.6 that equation (71) implies the Robertson uncertainty relation, equation (14). More precisely, we have shown that it only implies a special case of it, namely equation (96). Therefore, it is natural to conjecture that there exists a more general entropic uncertainty relation which implies the Robertson uncertainty relation (14) for any matrix $\mathbf{C}$, more general than in equation (97). Our first conjecture is an extension of equation (71) for $m$ variables:

Conjecture 1. Any $n$-modal state $\rho$ satisfies the entropic uncertainty relation

$h\left(R_{1}\right)+h\left(R_{2}\right)+\cdots+h\left(R_{m}\right)-\frac{1}{2} \ln \left(\frac{\sigma_{1}^{2} \sigma_{2}^{2} \cdots \sigma_{m}^{2}}{\operatorname{det} \Gamma}\right) \geqslant \frac{1}{2} \ln \left((2 \pi e)^{m} \operatorname{det} \mathbf{C}\right)$

where $\hat{R}_{i}$ 's are $m$ arbitrary continuous observables, $\sigma_{i}^{2}$ is the variance of each $\hat{R}_{i}$ while $\Gamma$ is the covariance matrix of the $\hat{R}_{i}$ 's, and $\mathbf{C}$ is the matrix of commutators. The elements of $\Gamma$ and $\mathbf{C}$ are defined as in equation (15).

This entropic uncertainty relation is valid regardless of whether the $\hat{R}_{i}$ 's commute or not, but is interesting for an even number of them only. Indeed, as mentioned for the Robertson uncertainty relation, equation (14), when $m$ is odd, $\operatorname{det} \mathbf{C}=0$ and the lower bound in conjecture 1 equals $-\infty$. Note also that equation (99) is defined only when $m \leqslant 2 n$, where $n$ is the number of modes of the state $\rho$. Indeed, if $m>2 n$, the determinant of the covariance matrix $\Gamma$ vanishes (we can always write one column as a linear combination of two other columns). This is consistent with the fact that $\operatorname{det} \mathbf{C}$ vanishes in this case too. Indeed, $\operatorname{det} \mathbf{C} \geqslant 0$ since $\mathbf{C}$ is an anti-symmetric matrix [40], so that equation (14) implies that if $\operatorname{det} \boldsymbol{\Gamma}$ is null, so is $\operatorname{det} \mathbf{C}$.

Finally, let us mention that equation (99) is invariant under the scaling of one variable. Assume, with no loss of generality, that $R_{1} \rightarrow R_{1}^{\prime}=a R_{1}$, where $a$ is some scaling constant. Then, the entropy is transformed into $h\left(R_{1}^{\prime}\right)=h\left(R_{1}\right)+\ln |a|$, the variance becomes $\sigma_{1^{\prime}}^{2}=a^{2} \sigma_{1}^{2}$, the covariances $\boldsymbol{\Gamma}_{1^{\prime} j}=a \boldsymbol{\Gamma}_{1 j}$, and the commutators $\left[R_{1}^{\prime}, R_{j}\right]=a\left[R_{1}, R_{j}\right]$. This implies that both $\boldsymbol{\Gamma}$ and $\mathbf{C}$ have one column and one row multiplied by $a$, so that $\operatorname{det} \Gamma$ and $\operatorname{det} \mathbf{C}$ are both multiplied by $a^{2}$. Inserting these new values in equation (99), we see that the constant term $\ln |a|$ appears on both sides of the inequality, confirming the invariance of this entropic uncertainty relation.

It is straightforward to prove the validity of equation (99) for Gaussian states. Inserting the entropy of Gaussian-distributed variable $h\left(R_{i}\right)=\ln \left(2 \pi e \sigma_{i}^{2}\right) / 2$ into equation (99), we obtain

$$
\frac{1}{2} \ln \left((2 \pi e)^{m} \operatorname{det} \Gamma\right) \geqslant \frac{1}{2} \ln \left((2 \pi e)^{m} \operatorname{det} \mathbf{C}\right) \Leftrightarrow \operatorname{det} \Gamma \geqslant \operatorname{det} \mathbf{C}
$$

which is nothing else but the Robertson uncertainty relation, equation (14).

The difficult (unresolved) problem is to prove this conjecture for any state, not necessarily Gaussian. Here, we restrict ourselves to show that, for any state, equation (99) implies the Robertson uncertainty relation (14) in its general form. The method works as usual. First, we use the entropy power of each variable $R_{i}$ 


$$
N_{i}=\frac{1}{2 \pi e} \mathrm{e}^{2 h\left(R_{i}\right)}
$$

to rewrite equation (99) into its entropy-power form

$$
N_{1} N_{2} \cdots N_{m} \frac{\operatorname{det} \Gamma}{\sigma_{1}^{2} \sigma_{2}^{2} \cdots \sigma_{m}^{2}} \geqslant \operatorname{det} \mathbf{C} \text {. }
$$

Then, we use the fact that, for a fixed variance, the maximum entropy is given by a Gaussian distribution, that is, $N_{i} \leqslant \sigma_{i}^{2}$. We thus obtain the chain of inequalities

$$
\operatorname{det} \Gamma \geqslant N_{1} N_{2} \cdots N_{m} \frac{\operatorname{det} \Gamma}{\sigma_{1}^{2} \sigma_{2}^{2} \cdots \sigma_{m}^{2}} \geqslant \operatorname{det} \mathbf{C}
$$

from which we deduce equation (14).

Now, in order to avoid the problem that the entropic uncertainty relation (99) is only defined for $m \leqslant 2 n$, we may relax the bound by ignoring the correlations between the $R_{i}$ 's as characterized by $\boldsymbol{\Gamma}$. This leads to the following (weaker) relation:

Conjecture 2. Any $n$-modal state $\rho$ satisfies the entropic uncertainty relation

$$
h\left(R_{1}\right)+h\left(R_{2}\right)+\cdots+h\left(R_{m}\right) \geqslant \frac{1}{2} \ln \left((2 \pi e)^{m} \operatorname{det} \mathbf{C}\right)
$$

where the $R_{i}$ 's are $m$ arbitrary continuous observables and $\mathbf{C}$ is the matrix of commutators as defined in equation (15).

This entropic uncertainty relation may probably be easier to prove than equation (99). From its entropy-power formulation, we see that it implies the weaker form of the Robertson uncertainty relation, equation (38), that is, we have the chain of inequalities

$$
\sigma_{1}^{2} \sigma_{2}^{2} \cdots \sigma_{m}^{2} \geqslant N_{1} N_{2} \cdots N_{m} \geqslant \operatorname{det} \mathbf{C} .
$$

It is also immediate to see that equation (99) implies equation (104) as a result of Hadamard inequality, equation (37), so that conjecture 2 is indeed weaker than conjecture 1.

A problem with these two conjectures remains that they are irrelevant for an odd number $m$ of observables. We then conjecture a third version of an entropic uncertainty relation which holds for any $m$, but only for one-mode states $(n=1)$ :

Conjecture 3. Let $\mathbf{R}=\left(\hat{R}_{1}, \cdots, \hat{R}_{m}\right)$ be a vector of $m$ continuous observables acting on one mode as $\mathbf{R}=\mathbf{a} \hat{x}+\mathbf{b} \hat{p}$, with $\hat{x}$ and $\hat{p}$ being the canonically conjugate quadratures of the mode as in equation (19). Then, any one-mode state $\rho$ satisfies the entropic uncertainty relation

$$
h\left(R_{1}\right)+h\left(R_{2}\right)+\cdots+h\left(R_{m}\right) \geqslant \frac{m}{2} \ln \left(\frac{2 \pi e}{m}|\mathbf{a} \wedge \mathbf{b}|\right)
$$

where the norm of the wedge product between vectors $\mathbf{a}$ and $\mathbf{b}$ is computed with equation (20).

Its entropy-power form is

$$
N_{1} N_{2} \cdots N_{m} \geqslant\left(\frac{|\mathbf{a} \wedge \mathbf{b}|}{m}\right)^{m}
$$


where the $N_{i}$ are defined as in equation (101). From equation (107), we can deduce the variance-based uncertainty relation equation (18), which was derived in [31].

Let us mention that, for $m=2$, conjecture 3 reduces to the entropic uncertainty relation (59) for two arbitrary quadratures in the special case of one mode $(n=1)$, so it is proven [55]. Indeed, if $\hat{R}_{1}=a_{1} \hat{x}+b_{1} \hat{p}$ and $\hat{R}_{2}=a_{2} \hat{x}+b_{2} \hat{p}$, we have

$$
|\mathbf{a} \wedge \mathbf{b}|=\left|a_{1} b_{2}-a_{2} b_{1}\right|=\left|\left[\hat{R}_{1}, \hat{R}_{2}\right]\right|,
$$

so that equation (106) becomes identical to equation (59). Furthermore, conjecture 3 reduces to the entropic uncertainty relation (58) for two rotated quadratures, that is, when we choose $\mathbf{a}=(\cos \theta, \sin \theta)$ and $\mathbf{b}=(\cos \phi, \sin \phi)$, so that $|\mathbf{a} \wedge \mathbf{b}|=|\sin (\theta-\phi)|$.

Finally, let us consider the special case of equation (21), where we have $m$ quadratures that are equidistributed around the unit circle, that is

$$
\hat{R}_{i}=\cos \phi_{i} \hat{x}+\sin \phi_{i} \hat{p} \quad \text { with } \quad \phi_{i}=\frac{2 \pi(i-1)}{m}, \quad i=1, \ldots, m .
$$

In this case, conjecture 3 reduces to the following entropic uncertainty relation, which was already conjectured in [31], and which we prove here ${ }^{10}$, namely

Conjecture 4. Let $\mathbf{R}=\left(R_{1}, \cdots, R_{m}\right)$ be a vector of $m$ continuous observables acting on one mode and equidistributed as defined in equation (109). Then, any one-modal state $\rho$ satisfies the entropic uncertainty relation [31]

$$
h\left(R_{1}\right)+h\left(R_{2}\right)+\cdots+h\left(R_{m}\right) \geqslant \frac{m}{2} \ln (\pi e) .
$$

Indeed, for equidistributed $R_{i}$ 's, we have $|\mathbf{a} \wedge \mathbf{b}|=m / 2$ as shown in equation (24), so that equation (106) reduces to equation (110). Similarly as before, its entropy-power form is

$$
N_{1} N_{2} \cdots N_{m} \geqslant\left(\frac{1}{2}\right)^{m},
$$

where the $N_{i}$ are defined as in equation (101) and, from it, we can deduce the variance-based uncertainty relation (25) as derived in [31].

We present here a partial proof of conjecture 4 using a variational method, following the same lines as in section 4.4 , which is based on the extremization of our functional

$$
F(\rho)=h\left(R_{1}\right)+\cdots+h\left(R_{m}\right) .
$$

After proving that the vacuum state is a local extremum of $F(\rho)$, we will again assume that it is its global minimum. This assumption seems reasonable since the vacuum minimizes the corresponding variance-based uncertainty relation (25) as shown in [31]. Let us consider pure states $\rho=|\psi\rangle\langle\psi|$ first. Here too, our functional $F(\rho)$ is invariant under displacements, so we can restrict to states centered on the origin. Inserting the constraints of normalization and zero mean values, we want to solve $\frac{\partial J}{\partial\langle\psi|}=0$, where

$$
J=h\left(R_{1}\right)+\cdots+h\left(R_{m}\right)+\lambda(\langle\psi \mid \psi\rangle-1)+\sum_{i=1}^{m} \mu_{i}\left\langle\psi\left|\hat{R}_{i}\right| \psi_{i}\right\rangle
$$

and $\lambda$ and $\mu_{i}$ are Lagrange multipliers. As shown in section 4.4,

\footnotetext{
${ }^{10}$ The proof is conditional on one reasonable assumption, see below.
} 


$$
\begin{aligned}
& \frac{\partial h\left(R_{i}\right)}{\partial\langle\psi|}=-\left(\ln P\left(R_{i}\right)+1\right)|\psi\rangle \\
& \frac{\partial}{\partial\langle\psi|}\left(\lambda(\langle\psi \mid \psi\rangle-1)+\sum_{i=1}^{m} \mu_{i}\left\langle\psi\left|\hat{R}_{i}\right| \psi\right\rangle\right)=\left(\lambda+\sum_{i=1}^{m} \mu_{i} \hat{R}_{i}\right)|\psi\rangle
\end{aligned}
$$

so that the variational equation becomes

$$
\left[-\ln \left(P\left(R_{1}\right) P\left(R_{2}\right) \cdots P\left(R_{m}\right)\right)-m+\lambda+\sum_{i=1}^{m} \mu_{i} \hat{R}_{i}\right]|\psi\rangle=0 .
$$

Thus, the eigenstates of equation (115) are the states extremizing the uncertainty functional. As before, instead of looking for eigenstates, we check that the vacuum state $|0\rangle$ is a solution of equation (115). It means that $P\left(R_{i}\right)$ is a Gaussian distribution

$$
P\left(\hat{R}_{i}\right)=\frac{1}{\sqrt{\pi}} \mathrm{e}^{-R_{i}^{2}}
$$

where we used equation (33) and the fact that the variance of $\hat{R}_{i}$ in the vacuum state is $1 / 2$ for all $\hat{R}_{i}$ 's of equation (109). The variational equation can now be written as

$$
\left[\frac{m}{2} \ln (\pi)+\sum_{i=1}^{m} \hat{R}_{i}^{2}-m+\lambda+\sum_{i=1}^{m} \mu_{i} \hat{R}_{i}\right]|0\rangle=0
$$

which can be further simplified as

$$
\left[\frac{m}{2} \ln (\pi)-\frac{m}{2}+\lambda+\sum_{i=1}^{m} \mu_{i} \hat{R}_{i}\right]|0\rangle=0
$$

by using the fact that

$$
\begin{aligned}
\sum_{i=1}^{m} \hat{R}_{i}^{2}|\psi\rangle & =\sum_{i=1}^{m}\left[\cos \left(\frac{2 \pi(i-1)}{m}\right) \hat{x}+\sin \left(\frac{2 \pi(i-1)}{m}\right) \hat{p}\right]^{2}|0\rangle \\
& =\frac{m}{2}\left(\hat{x}^{2}+\hat{p}^{2}\right)|0\rangle \\
& =\frac{m}{2}|0\rangle .
\end{aligned}
$$

The value of $\lambda=\frac{m}{2} \ln (e / \pi)$ is found by multiplying equation (118) on the left by $\langle 0|$ and using the normalization condition as well as the fact that all mean values $\left\langle 0\left|\hat{R}_{i}\right| 0\right\rangle$ must vanish. We are then left with $\sum_{i=1}^{m} \mu_{i} \hat{R}_{i}|0\rangle=0$, which is satisfied if $\mu_{i}=0$ for all $i$. Thus, we have shown that there exists an appropriate choice of $\lambda$ and $\mu_{i}$ so that the vacuum extremizes the uncertainty functional. Assuming that it is the global minimizer, we have proved equation (110) for pure states since $F(|0\rangle)=\frac{m}{2} \ln (\pi e)$. Due to the concavity of the differential entropy, the entropic uncertainty relation (110) then holds for mixed states too. 
Table 1. Summary of the entropic uncertainty relations (UR) expressed in terms of differential entropies (first column), their corresponding entropy-power formulations (second column), as well as their implied variance-based uncertainty relations (third column). The symbol $\checkmark$ means that the entropic uncertainty relation is proven, $\dagger$ that it is proven conditionally on reasonable assumptions and $*$ that it is a conjecture. We set $\hbar=1$ in all equations.

\begin{tabular}{|c|c|c|c|}
\hline Entropic UR & & Entropy-power UR & Variance-based UR \\
\hline $\begin{array}{l}h(x)+h(p) \geqslant \ln (\pi e) \\
\text { Bialynicki-Birula and Mycielski for } n=1 \text {, equation (2) }\end{array}$ & $\checkmark$ & $\begin{array}{l}N_{x} N_{p} \geqslant \frac{1}{4} \\
\text { equation }(44)\end{array}$ & $\begin{array}{l}\sigma_{x}^{2} \sigma_{p}^{2} \geqslant \frac{1}{4} \\
\text { Heisenberg, equation (3) }\end{array}$ \\
\hline $\begin{array}{l}h(\mathbf{x})+h(\mathbf{p})-\frac{1}{2}\left(\frac{\operatorname{det} \gamma_{\mathbf{x}} \operatorname{det} \gamma_{\mathrm{p}}}{\operatorname{det} \gamma}\right) \geqslant n \ln (\pi e) \\
\text { equation (49) }\end{array}$ & $\dagger$ & $\begin{array}{l}N_{\mathbf{x}} N_{\mathbf{p}}\left(\frac{\operatorname{det} \gamma}{\operatorname{det} \gamma_{\mathbf{x}} \operatorname{det} \gamma_{\mathbf{p}}}\right){ }^{1 / n} \geqslant \frac{1}{4} \\
\text { equation }(55)\end{array}$ & $\begin{array}{l}\operatorname{det} \gamma \geqslant \frac{1}{4^{n}} \\
\text { equation }(13)\end{array}$ \\
\hline $\begin{array}{l}h(\mathbf{y})+h(\mathbf{z}) \geqslant \ln \left((\pi e)^{n}|\operatorname{det} \mathbf{K}|\right) \\
\text { equation }(62)\end{array}$ & $\checkmark$ & $\begin{array}{l}N_{\mathbf{y}} N_{\mathbf{z}} \geqslant \frac{|\operatorname{det} \mathbf{K}|^{2 / n}}{4} \\
\text { equation }(64)\end{array}$ & $\begin{array}{l}\operatorname{det} \boldsymbol{\Gamma}_{\mathbf{y}} \operatorname{det} \boldsymbol{\Gamma}_{\mathbf{z}} \geqslant \frac{|\operatorname{det} \mathbf{K}|^{2}}{4^{n}} \\
\text { equation }(65)\end{array}$ \\
\hline $\begin{array}{l}h(\mathbf{y})+h(\mathbf{z})-\frac{1}{2} \ln \left(\frac{\operatorname{det} \boldsymbol{\Gamma}_{\mathbf{y}} \operatorname{det} \boldsymbol{\Gamma}_{\mathbf{z}}}{\operatorname{det} \boldsymbol{\Gamma}}\right) \geqslant \ln \left((\pi e)^{n}|\operatorname{det} \mathbf{K}|\right) \\
\text { equation (71) }\end{array}$ & $\dagger$ & $\begin{array}{l}N_{\mathbf{y}} N_{\mathbf{z}}\left(\frac{\operatorname{det} \boldsymbol{\Gamma}}{\operatorname{det} \boldsymbol{\Gamma}_{\mathbf{y}} \operatorname{det} \boldsymbol{\Gamma}_{\mathbf{z}}}\right) 1 / n \geqslant \frac{|\operatorname{det} \mathbf{K}|^{2 / n}}{4} \\
\text { equation (95) }\end{array}$ & $\begin{array}{l}\operatorname{det} \boldsymbol{\Gamma} \geqslant \frac{|\operatorname{det} \mathbf{K}|^{2}}{4^{n}} \\
\text { equation }(96)\end{array}$ \\
\hline $\begin{array}{l}h\left(R_{1}\right)+\cdots+h\left(R_{m}\right)-\frac{1}{2} \ln \left(\frac{\sigma_{1}^{2} \sigma_{2}^{2} \cdots \sigma_{m}^{2}}{\operatorname{det} \boldsymbol{\Gamma}}\right) \geqslant \frac{1}{2} \ln \left((2 \pi e)^{m} \operatorname{det} \mathbf{C}\right) \\
\text { equation (99) }\end{array}$ & $*$ & $\begin{array}{l}N_{1} \cdots N_{m} \frac{\operatorname{det} \boldsymbol{\Gamma}}{\sigma_{1}^{2} \sigma_{2}^{2} \cdots \sigma_{m}^{2}} \geqslant \operatorname{det} \mathbf{C} \\
\text { equation }(102)\end{array}$ & $\begin{array}{l}\operatorname{det} \boldsymbol{\Gamma} \geqslant \operatorname{det} \mathbf{C} \\
\text { Robertson, equation }(14)\end{array}$ \\
\hline $\begin{array}{l}h\left(R_{1}\right)+\cdots+h\left(R_{m}\right) \geqslant \frac{1}{2} \ln \left((2 \pi e)^{m} \operatorname{det} \mathbf{C}\right) \\
\text { equation }(104)\end{array}$ & $*$ & $\begin{array}{l}N_{1} \cdots N_{m} \geqslant \operatorname{det} \mathbf{C} \\
\text { equation (105) }\end{array}$ & $\begin{array}{l}\sigma_{1}^{2} \cdots \sigma_{m}^{2} \geqslant \operatorname{det} \mathbf{C} \\
\text { Robertson, equation }(38)\end{array}$ \\
\hline $\begin{array}{l}h\left(R_{1}\right)+\cdots+h\left(R_{m}\right) \geqslant \frac{m}{2} \ln \left(\frac{2 \pi e}{m}|\mathbf{a} \wedge \mathbf{b}|\right) \\
\text { equation (106) }\end{array}$ & * & $\begin{array}{l}N_{1} \cdots N_{m} \geqslant\left(\frac{|\mathbf{a} \wedge \mathbf{b}|}{m}\right)^{m} \\
\text { equation (107) }\end{array}$ & $\begin{array}{l}\sigma_{1}^{2} \cdots \sigma_{m}^{2} \geqslant\left(\frac{|\mathbf{a} \wedge \mathbf{b}|}{m}\right)^{m} \\
\text { equation }(18)\end{array}$ \\
\hline $\begin{array}{l}h\left(R_{1}\right)+\cdots+h\left(R_{m}\right) \geqslant \frac{m}{2} \ln (\pi e) \\
\text { equation }(110)\end{array}$ & $\dagger$ & $\begin{array}{l}N_{1} \cdots N_{m} \geqslant \frac{1}{2^{m}} \\
\text { equation }(111)\end{array}$ & $\begin{array}{l}\sigma_{1}^{2} \cdots \sigma_{m}^{2} \geqslant \frac{1}{2^{m}} \\
\text { equation }(25)\end{array}$ \\
\hline
\end{tabular}




\section{Conclusion}

We have reviewed continuous-variable entropic uncertainty relations starting from the very first formulation by Hirschman and the proof by Bialynicki-Birula and Mycielski to the recent entropic uncertainty relation between non-canonically conjugate variables, whose lower bound depends on the determinant of a matrix of commutators. We then showed that, by taking correlations into account, it is possible to define an entropic uncertainty relation for any two vectors of intercommuting quadratures whose minimum-uncertainty states are all pure Gaussian states. Finally, we derived several conjectures for an entropic uncertainty relation addressing more than 2 continuous observables and gave a partial proof of one of them.

In table 1, we provide a summary of all entropic uncertainty relations (for Shannon differential entropies) encountered in this paper. These entropic uncertainty relations appear in the first column of table 1 . The symbol $\checkmark$ means that the relation is proven, $\dagger$ that it is proven conditionally on reasonable assumptions, and $*$ that it is still a conjecture. As emphasized throughout this paper, entropic uncertainty relations are conveniently formulated in terms of entropy powers. The corresponding entropy-power uncertainty relations are then shown in the second column of table 1. Further, using the fact that the maximum entropy for a fixed variance is reached by a Gaussian distribution, a variance-based uncertainty relation can easily be deduced from each entropy-power uncertainty relation. This is what is done in the third column of table 1, where we show the variance-based uncertainty relations that are implied by all entropy-power uncertainty relations.

We conclude this paper by noting that, although significant progress on entropic uncertainty relations has been achieved lately, we still lack a symplectic-invariant entropic uncertainty relation. All relations we have discussed are invariant under displacements (corresponding to a translation of the variables in phase space), and most of them are also invariant under squeezing transformations (corresponding to a scaling of the variables). However, no entropic uncertainty relation is invariant under rotations, which would make it invariant under the complete set of symplectic transformations. This is, however, a natural property of many variancebased uncertainty relations, such as the Robertson-Schrödinger relation (8). The invariance of the determinant of $\gamma$ makes the latter relation invariant under all symplectic transformations, hence under all Gaussian unitaries (since it is invariant under displacements too). A mentioned in section 3.4 , the joint entropy $h(x, p)$ would have the desired property to build a symplecticinvariant entropic uncertainty relation, but it is not defined for states with a negative Wigner function (see also [9, 39]). The tight entropic uncertainty relations of equations (49) and (71) admit all pure Gaussian states as minimum-uncertainty state, but are nevertheless not invariant under rotations. A recent attempt at defining a symplectic-invariant entropic uncertainty relation is made in [65], which builds on a multi-copy uncertainty observable that is related to the Schwinger representation of a spin state via harmonic oscillators.

\section{Acknowledgments}

We thank Michael Jabbour and Luc Vanbever for their collaboration and Stefan Weigert for helpful discussions. This work was supported by the FRS-FNRS Foundation under Projects No. T.0199.13 and T.0224.18. AH acknowledges financial support from the FRS-FNRS Foundation. 


\section{Appendix A. Symplectic formalism}

Here, we briefly review the representation of Gaussian states and unitaries in phase space based on the symplectic formalism (see also [37]). A $n$-mode Gaussian state $\rho$ has a Gaussian Wigner function of the form

$$
W_{\mathrm{G}}(\mathbf{x}, \mathbf{p})=\frac{1}{(2 \pi)^{n} \sqrt{\operatorname{det} \gamma}} \mathrm{e}^{-\frac{1}{2}(\mathbf{r}-\langle\mathbf{r}\rangle)^{T} \gamma^{-1}(\mathbf{r}-\langle\mathbf{r}\rangle)}
$$

and is completely characterized by its vector of mean values $\langle\mathbf{r}\rangle=\operatorname{tr}(\mathbf{r} \rho)$ and its covariance matrix $\gamma$, whose elements are given by

$$
\gamma_{i j}=\frac{1}{2}\left\langle\left\{\hat{r}_{i}, \hat{r}_{j}\right\}\right\rangle-\left\langle\hat{r}_{i}\right\rangle\left\langle\hat{r}_{j}\right\rangle .
$$

Here, $\mathbf{r}=\left(\hat{x}_{1}, \hat{p}_{1}, \hat{x}_{2}, \hat{p}_{2}, \cdots, \hat{x}_{n}, \hat{p}_{n}\right)$ is the quadrature vector, $\langle\cdot\rangle$ stands for the expectation value $\operatorname{tr}(\cdot \rho)$, and $\{\cdot, \cdot\}$ stands for the anti-commutator. Remark that the covariance matrix $\gamma$ is a real, symmetric, and positive semi-definite matrix. It must also comply with the uncertainty relation

$$
\gamma+\mathrm{i} \frac{\Omega}{2} \geqslant 0
$$

where $\Omega$ is the so-called symplectic form, defined as

$$
\Omega=\bigoplus_{k=1}^{n} \omega, \quad \omega=\left(\begin{array}{cc}
0 & 1 \\
-1 & 0
\end{array}\right) .
$$

Equation (A.3) is a necessary and sufficient condition that $\gamma$ has to fulfill in order to be the covariance matrix of a physical state [66].

The purity $\mu$ of a Gaussian state is given by

$$
\mu_{\mathrm{G}}=\frac{1}{2^{n} \sqrt{\operatorname{det} \gamma}}
$$

and it can be shown that pure states having $\operatorname{det} \gamma=1 / 4^{n}$ are necessarily Gaussian.

The simplest example of a one-modal Gaussian state is the vacuum state $|0\rangle$. It has a vector of mean values equal to $(0,0)^{\mathrm{T}}$ and its covariance matrix is given by $\gamma_{v a c}=1 / 2$. We can displace the vacuum in phase state by applying a Gaussian unitary resulting in another Gaussian state called a coherent state $|\alpha\rangle=D(\alpha)|0\rangle$, where $D(\alpha)=\mathrm{e}^{\alpha \hat{a}^{\dagger}-\alpha^{*} \hat{a}}$ and $\hat{a}$ is the annihilation operator. The covariance matrix of a coherent state is the same as for the vacuum, but its vector of mean values changes as $\langle\mathbf{r}\rangle_{\alpha}=\sqrt{2}\left(\begin{array}{l}\Re(\alpha) \\ \Im(\alpha)\end{array}\right)$. As another Gaussian unitary, we can squeeze the variance of a quadrature and obtain another Gaussian state known as a squeezed state $|z\rangle=S(z)|0\rangle$ with $S(z)=\mathrm{e}^{\frac{1}{2}\left(z^{*} \hat{a}^{2}-z \hat{a}^{\dagger 2}\right)}$, where $z=r \mathrm{e}^{\mathrm{i} \phi}$ is a complex number ( $r$ is the squeezing parameter and $\phi$ the squeezing angle). The symplectic matrix associated to this Gaussian unitary is given by

$$
\mathcal{S}_{z}=\left(\begin{array}{cc}
\cosh r-\cos 2 \phi \sinh r & -\sin 2 \phi \sinh r \\
-\sin 2 \phi \sinh r & \cosh r+\cos 2 \phi \sinh r
\end{array}\right)
$$

so that the covariance matrix of a squeezed state is given by

$$
\gamma_{z}=\mathcal{S}_{z} \gamma_{v a c} \mathcal{S}_{z}^{T}=\frac{1}{2}\left(\begin{array}{cc}
\cosh 2 r-\cos 2 \phi \sinh 2 r & -\sin 2 \phi \sinh 2 r \\
-\sin 2 \phi \sinh 2 r & \cosh 2 r+\cos 2 \phi \sinh 2 r
\end{array}\right) .
$$


A squeezing in the $x(p)$ direction corresponds to the choice $\phi=0(\phi=\pi / 2)$. Yet another (one-mode) Gaussian operation is the phase-shift $R(\theta)=\mathrm{e}^{-\mathrm{i} \theta \hat{a}^{\dagger} \hat{a}}$. Its associated symplectic matrix is simply given by the rotation matrix

$$
\mathcal{R}_{\theta}=\left(\begin{array}{cc}
\cos \theta & \sin \theta \\
-\sin \theta & \cos \theta
\end{array}\right)
$$

The above operations are all the possible one-modal Gaussian unitaries. For $n$ modes, there is a larger set of Gaussian unitary operations, which we do not need to discuss here. The key point is that, in state space, a Gaussian unitary always transforms a Gaussian state onto a Gaussian state. Its corresponding action in phase space is expressed via a symplectic transformation. That is, if a Gaussian unitary $U$ transforms $\rho$ according to

$$
\hat{\rho} \rightarrow U \hat{\rho} U^{\dagger}
$$

its quadratures in phase space are transformed as

$$
\hat{\mathbf{r}} \rightarrow \mathcal{S} \hat{\mathbf{r}}+\mathbf{d}
$$

where $\mathbf{d}$ is a real vector of dimension $2 n$ and $\mathcal{S}$ is a real $2 n \times 2 n$ matrix. Regarding the mean values and covariance matrix of $\rho$, the transformation rules are

$$
\langle\mathbf{r}\rangle \rightarrow \mathcal{S}\langle\mathbf{r}\rangle+\mathbf{d} \quad \text { and } \quad \gamma \rightarrow \mathcal{S} \gamma \mathcal{S}^{T}
$$

The commutation relations between the quadratures have to be preserved along this transformation, which is the case if the matrix $\mathcal{S}$ is symplectic, that is, if

$$
\mathcal{S} \Omega \mathcal{S}^{T}=\Omega
$$

where $\Omega$ is defined in equation (A.4). Note that $\Omega^{T}=\Omega^{-1}=-\Omega$ and $\Omega^{2}=-1$. Be aware that this definition of symplectic matrices is linked to the definition of $\mathbf{r}$ (i.e. the ordering of the entries in $\mathbf{r})$. If one chooses instead to define $\mathbf{r}=\left(\hat{x}_{1}, \cdots, \hat{x}_{n}, \hat{p}_{1}, \cdots, \hat{p}_{n}\right)$, then the matrix $\mathcal{S}$ is symplectic if $\mathcal{S} J \mathcal{S}^{T}=J$ with $J=\left(\begin{array}{cc}0 & \mathbb{1} \\ -\mathbb{1} & 0\end{array}\right)$. Here too, $J^{T}=J^{-1}=-J$ and $J^{2}=-\mathbb{1}$.

In addition, any symplectic matrix $\mathcal{S}$ has the following properties:

- The matrices $\mathcal{S}^{T}, \mathcal{S}^{-1}$ and $-\mathcal{S}$ are also symplectic.

- The inverse of $\mathcal{S}$ is given by $\mathcal{S}^{-1}=-\Omega \mathcal{S}^{T} \Omega$ (or $\mathcal{S}^{-1}=-J \mathcal{S}^{T} J$, depending on the definition of $\mathbf{r}$ ).

- $\operatorname{det} \mathcal{S}=1$, which implies that $\operatorname{det} \gamma$ is conserved by any symplectic transformation.

- If $\mathbf{r}=\left(\hat{x}_{1}, \cdots, \hat{x}_{n}, \hat{p}_{1}, \cdots, \hat{p}_{n}\right)$ and $\mathcal{S}=\left(\begin{array}{ll}a & b \\ c & d\end{array}\right)$, then $\mathcal{S} J \mathcal{S}^{T}=J$ implies that $a b^{T}$ and $c d^{T}$ are symmetric matrices and $a d^{T}-b c^{T}=\mathbb{1}$.

- In terms of the associated symplectic transformation, a Gaussian unitary will be passive (it conserves the mean photon number) if and only if

$$
\mathbf{d}=0 \quad \text { and } \quad \mathcal{S}^{T} \mathcal{S}=\mathbb{1},
$$

which means that the symplectic matrix $\mathcal{S}$ must be orthogonal.

The Williamson's theorem [67] states that, after the appropriate symplectic transformation, every real, positive semidefinite matrix of even dimension can be brought to a diagonal form $\gamma^{\oplus}$, with its symplectic values $\nu_{k}$ on the diagonal (each $\nu_{k}$ is doubly degenerate). In other words, there exists a symplectic matrix $\mathcal{S}$ such that ${ }^{11}$

\footnotetext{
${ }^{11}$ We use here the definition $\mathbf{r}=\left(\hat{x}_{1}, \hat{p}_{1}, \cdots, \hat{x}_{n}, \hat{p}_{n}\right)$.
} 


$$
\gamma=\mathcal{S} \gamma^{\oplus} \mathcal{S}^{T}, \quad \text { where } \quad \gamma^{\oplus}=\bigoplus_{k=1}^{n} \nu_{k} \mathbb{1}_{2 \times 2} .
$$

Obviously, since the determinant of a symplectic matrix is equal to $1, \gamma$ and $\gamma^{\oplus}$ have the same determinant. Therefore, for a one-mode state, its symplectic value is simply equal to $\sqrt{\operatorname{det} \gamma}$. For a two-mode state, the two symplectic values $\nu_{ \pm}$can be found using the following formula [68]

$$
\nu_{ \pm}=\sqrt{\frac{\Delta \pm \sqrt{\Delta^{2}-4 \operatorname{det} \gamma}}{2}}
$$

where the covariance can be written in the block form

$$
\gamma=\left(\begin{array}{cc}
A & C \\
C^{T} & B
\end{array}\right)
$$

and $\Delta=|A|+|B|+2|C|$. In general, one can find the symplectic values by diagonalizing the matrix $i \Omega \gamma$ and taking the absolute value of its eigenvalues (see e.g. [37, 39]).

\section{Appendix B. Calculation of $\operatorname{det} \Gamma$}

Here, we compute the determinant of the covariance matrix $\boldsymbol{\Gamma}$ of the $y, z$-quadratures (see equation (70)) as needed in the evaluation of the tight entropic uncertainty relation for two arbitrary vectors of quadratures, equation (71). As before, let $\mathbf{y}=\left(\hat{y}_{1}, \cdots \hat{y}_{n}\right)^{T}$ be a vector of commuting quadratures and $\mathbf{z}=\left(\hat{z}_{1}, \cdots \hat{z}_{n}\right)^{T}$ be another vector of commuting quadratures. We now suppose that they correspond to the output $x$-quadratures after applying two symplectic transformations denoted as $\mathcal{A}$ and $\mathcal{B}$ onto the $2 n$-dimensional vector of input quadratures $\mathbf{r}=\left(\hat{x}_{1}, \cdots, \hat{x}_{n}, \hat{p}_{1}, \cdots, \hat{p}_{n}\right)^{T}$. The corresponding $2 n$-dimensional vectors of output quadratures are written as

$$
\mathbf{r}_{A}=\mathcal{A} \mathbf{r} \equiv\left(\begin{array}{l}
\mathbf{y} \\
\mathbf{q}
\end{array}\right), \quad \mathbf{r}_{B}=\mathcal{B} \mathbf{r} \equiv\left(\begin{array}{l}
\mathbf{z} \\
\mathbf{o}
\end{array}\right)
$$

where $\mathbf{q}$ (resp. o) is the vector of quadratures that are canonically conjugate with $\mathbf{y}$ (resp. $\mathbf{z}$ ). Since equation (B.1) tells us how to obtain $\mathbf{y}$ and $\mathbf{z}$ from $\mathbf{x}$ and $\mathbf{p}$ through the symplectic transformations $\mathcal{A}$ and $\mathcal{B}$, we can compute the elements of the covariance matrix $\Gamma$ for the $y, z$ quadratures, namely

$$
\Gamma_{i j}=\frac{1}{2}\left\langle\hat{R}_{i} \hat{R}_{j}+\hat{R}_{j} \hat{R}_{i}\right\rangle-\left\langle\hat{R}_{i}\right\rangle\left\langle\hat{R}_{j}\right\rangle
$$

with $\mathbf{R}=\left(\hat{y}_{1}, \ldots, \hat{y}_{n}, \hat{z}_{1}, \ldots, \hat{z}_{n}\right)^{T}$. For example, we may evaluate $\boldsymbol{\Gamma}_{i j}$ for $1 \leqslant i, j \leqslant n$, namely

$$
\begin{aligned}
\boldsymbol{\Gamma}_{i j} & =\frac{1}{2}\left\langle\sum_{k=1}^{2 n} \mathcal{A}_{i k} \hat{r}_{k} \sum_{l=1}^{2 n} \mathcal{A}_{j l} \hat{r}_{l}+\sum_{l=1}^{2 n} \mathcal{A}_{j l} \hat{r}_{l} \sum_{k=1}^{2 n} \mathcal{A}_{i k} \hat{r}_{k}\right\rangle-\left\langle\sum_{k=1}^{2 n} \mathcal{A}_{i k} \hat{r}_{k}\right\rangle\left\langle\sum_{l=1}^{2 n} \mathcal{A}_{j l} \hat{r}_{l}\right\rangle \\
& =\sum_{k=1}^{2 n} \sum_{l=1}^{2 n} \mathcal{A}_{i k} \mathcal{A}_{j l} \gamma_{k l} \\
& =\sum_{k=1}^{2 n} \sum_{l=1}^{2 n} \mathcal{A}_{i k} \gamma_{k l} \mathcal{A}_{l j}^{T} \\
& =\left(\mathcal{A} \gamma \mathcal{A}^{T}\right)_{i j} .
\end{aligned}
$$


Similarly, we can show that $\boldsymbol{\Gamma}_{i+n, j+n}=\left(\mathcal{B} \gamma \mathcal{B}^{T}\right)_{i j}$ and $\boldsymbol{\Gamma}_{i, j+n}=\left(\mathcal{A} \gamma \mathcal{B}^{T}\right)_{i j}$ for $1 \leqslant i, j \leqslant n$. Since the covariance matrix is symmetric, we obtain

$$
\boldsymbol{\Gamma}=\left(\begin{array}{cc}
\boldsymbol{\Gamma}_{\mathbf{y}} & \boldsymbol{\Gamma}_{y z} \\
\boldsymbol{\Gamma}_{y z} & \boldsymbol{\Gamma}_{\mathbf{z}}
\end{array}\right)=\left(\begin{array}{cc}
\left(\mathcal{A} \gamma \mathcal{A}^{T}\right)_{i, j=1, \ldots, n} & \left(\mathcal{A} \gamma \mathcal{B}^{T}\right)_{i, j=1, \ldots, n} \\
\left(\mathcal{B} \gamma \mathcal{A}^{T}\right)_{i, j=1, \ldots, n} & \left(\mathcal{B} \gamma \mathcal{B}^{T}\right)_{i, j=1, \ldots, n}
\end{array}\right) .
$$

Notice that matrices $\mathcal{A} \gamma \mathcal{A}^{T}, \mathcal{A} \gamma \mathcal{B}^{T}, \mathcal{B} \gamma \mathcal{A}^{T}$ and $\mathcal{B} \gamma \mathcal{B}^{T}$ all have dimensions $2 n \times 2 n$ but we truncate them to keep only the reduced matrices with indices running from 1 to $n$. Therefore, $\boldsymbol{\Gamma}_{\mathbf{y}}, \boldsymbol{\Gamma}_{\mathbf{z}}$ and $\boldsymbol{\Gamma}_{y z}$ have dimension $n \times n$, while $\boldsymbol{\Gamma}$ is a $2 n \times 2 n$ matrix.

To simplify the expression of $\boldsymbol{\Gamma}$, we use a block matrix representation of the symplectic transformations,

$$
\mathcal{A}=\left(\begin{array}{ll}
\mathcal{A}_{a} & \mathcal{A}_{b} \\
\mathcal{A}_{c} & \mathcal{A}_{d}
\end{array}\right) \quad \text { and } \quad \mathcal{B}=\left(\begin{array}{ll}
\mathcal{B}_{a} & \mathcal{B}_{b} \\
\mathcal{B}_{c} & \mathcal{B}_{d}
\end{array}\right)
$$

so that, for example,

$$
\begin{aligned}
\mathcal{A} \gamma \mathcal{A}^{T} & =\left(\begin{array}{ll}
\mathcal{A}_{a} & \mathcal{A}_{b} \\
\mathcal{A}_{c} & \mathcal{A}_{d}
\end{array}\right)\left(\begin{array}{cc}
\gamma_{\mathbf{x}} & \gamma_{\mathbf{x p}} \\
\gamma_{\mathbf{x p}} & \gamma_{\mathbf{p}}
\end{array}\right)\left(\begin{array}{cc}
\mathcal{A}_{a}^{T} & \mathcal{A}_{c}^{T} \\
\mathcal{A}_{b}^{T} & \mathcal{A}_{d}^{T}
\end{array}\right) \\
& =\left(\begin{array}{cc}
\mathcal{A}_{a} \gamma_{\mathbf{x}} \mathcal{A}_{a}^{T}+\mathcal{A}_{a} \gamma_{\mathbf{x p}} \mathcal{A}_{b}^{T}+\mathcal{A}_{b} \gamma_{\mathbf{x p}} \mathcal{A}_{a}^{T}+\mathcal{A}_{b} \gamma_{\mathbf{p}} \mathcal{A}_{b}^{T} & \ldots \\
\ldots & \ldots
\end{array}\right)
\end{aligned}
$$

where we do not need to express the matrix elements denoted with dots since all we need to compute is

$$
\left(\mathcal{A} \gamma \mathcal{A}^{T}\right)_{i, j=1, \ldots, n}=\mathcal{A}_{a} \gamma_{\mathbf{x}} \mathcal{A}_{a}^{T}+\mathcal{A}_{a} \gamma_{\mathbf{x p}} \mathcal{A}_{b}^{T}+\mathcal{A}_{b} \gamma_{\mathbf{x p}} \mathcal{A}_{a}^{T}+\mathcal{A}_{b} \gamma_{\mathbf{p}} \mathcal{A}_{b}^{T}
$$

By doing the same calculation for the other blocs of matrix $\boldsymbol{\Gamma}$, we obtain that it can be written as the product of three matrices,

$$
\boldsymbol{\Gamma}=\left(\begin{array}{cc}
\mathcal{A}_{a} & \mathcal{A}_{b} \\
\mathcal{B}_{a} & \mathcal{B}_{b}
\end{array}\right)\left(\begin{array}{cc}
\gamma_{\mathbf{x}} & \gamma_{\mathbf{x p}} \\
\gamma_{\mathbf{x p}} & \gamma_{\mathbf{p}}
\end{array}\right)\left(\begin{array}{cc}
\mathcal{A}_{a} & \mathcal{A}_{b} \\
\mathcal{B}_{a} & \mathcal{B}_{b}
\end{array}\right)^{T}
$$

In particular, the determinant of $\boldsymbol{\Gamma}$ is given by

$$
\operatorname{det} \boldsymbol{\Gamma}=\operatorname{det} \gamma\left[\operatorname{det}\left(\begin{array}{cc}
\mathcal{A}_{a} & \mathcal{A}_{b} \\
\mathcal{B}_{a} & \mathcal{B}_{b}
\end{array}\right)\right]^{2}
$$

Note that for a block matrix $\mathbf{M}$ of size $(n+m) \times(n+m)$ written as

$$
\mathbf{M}=\left(\begin{array}{cc}
\mathbf{A}_{n \times n} & \mathbf{B}_{n \times m} \\
\mathbf{C}_{m \times n} & \mathbf{D}_{m \times m}
\end{array}\right),
$$

it is easy to see that the following equality holds (assuming that $\mathbf{D}$ is invertible ${ }^{12}$ )

$$
\left(\begin{array}{ll}
\mathbf{A} & \mathbf{B} \\
\mathbf{C} & \mathbf{D}
\end{array}\right)\left(\begin{array}{cc}
\mathbb{1} & 0 \\
-\mathbf{D}^{-1} \mathbf{C} & \mathbb{1}
\end{array}\right)=\left(\begin{array}{cc}
\mathbf{A}-\mathbf{B D}^{-1} \mathbf{C} & \mathbf{B} \\
0 & \mathbf{D}
\end{array}\right) .
$$

Thus, the determinant of this equation is

$$
\operatorname{det}(\mathbf{M})=\operatorname{det}\left(\mathbf{A}-\mathbf{B D}^{-1} \mathbf{C}\right) \operatorname{det}(\mathbf{D})
$$

where we have exploited the fact that the determinant of a block triangular matrix is given by the product of the determinants of its diagonal blocks [69].

${ }^{12}$ If $\mathbf{D}$ is not invertible, equation (B.11) can be written in a similar way in terms of $\mathbf{A}^{-1}$. 
Moreover, since $\mathcal{B}$ represents a symplectic transformation, it hence satisfies $\mathcal{B}\left(\begin{array}{cc}0 & \mathbb{1} \\ -\mathbb{1} & 0\end{array}\right) \mathcal{B}^{T}=\left(\begin{array}{cc}0 & \mathbb{1} \\ -\mathbb{1} & 0\end{array}\right)$. In particular, this means that ${ }^{13} \mathcal{B}_{a} \mathcal{B}_{b}^{T}=\mathcal{B}_{b} \mathcal{B}_{a}^{T}$ or $\mathcal{B}_{a}=\mathcal{B}_{b} \mathcal{B}_{a}^{T} \mathcal{B}_{b}^{-T}$. Thus, using equation (B.12) together with the symmetry of the matrix $\mathcal{B}_{a} \mathcal{B}_{b}^{T}$, we can compute the determinant of the bloc matrix $\mathbf{M}$ in our case

$$
\begin{aligned}
\operatorname{det}\left[\left(\begin{array}{cc}
\mathcal{A}_{a} & \mathcal{A}_{b} \\
\mathcal{B}_{a} & \mathcal{B}_{b}
\end{array}\right)\right] & =\operatorname{det}\left(\mathcal{A}_{a}-\mathcal{A}_{b} \mathcal{B}_{b}^{-1} \mathcal{B}_{a}\right) \operatorname{det} \mathcal{B}_{b} \\
& =\operatorname{det}\left(\mathcal{A}_{a}-\mathcal{A}_{b} \mathcal{B}_{b}^{-1} \mathcal{B}_{b} \mathcal{B}_{a}^{T} \mathcal{B}_{b}^{-T}\right) \operatorname{det} \mathcal{B}_{b}^{T} \\
& =\operatorname{det}\left(\mathcal{A}_{a} \mathcal{B}_{b}^{T}-\mathcal{A}_{b} \mathcal{B}_{a}^{T}\right) \\
& =\operatorname{det}\left(\mathcal{B}_{b} \mathcal{A}_{a}^{T}-\mathcal{B}_{a} \mathcal{A}_{b}^{T}\right) .
\end{aligned}
$$

Thus, the determinant of $\boldsymbol{\Gamma}$ can be written in terms of the blocks composing the two symplectic transformations $\mathcal{A}$ and $\mathcal{B}$

$$
\operatorname{det} \boldsymbol{\Gamma}=\operatorname{det} \gamma\left(\operatorname{det}\left(\mathcal{B}_{b} \mathcal{A}_{a}^{T}-\mathcal{B}_{a} \mathcal{A}_{b}^{T}\right)\right)^{2}
$$

Now, this expression can be rewritten in a form that does not explicitly include the blocks composing $\mathcal{A}$ and $\mathcal{B}$ but uses the commutator matrix $\mathbf{K}$ instead. The elements of $\mathbf{K}$ are expressed as

$$
\begin{aligned}
\mathbf{K}_{j i} & =\left[\hat{y}_{j}, \hat{z}_{i}\right] \\
& =\sum_{k=1}^{2 n} \sum_{m=1}^{2 n} \mathcal{A}_{j k} \mathcal{B}_{i m}\left[\hat{r}_{k}, \hat{r}_{m}\right] \\
& =\mathrm{i} \sum_{m=1}^{2 n}\left(\sum_{k=1}^{n} \mathcal{A}_{j k} \mathcal{B}_{i m} \delta_{m, k+n}-\sum_{k=n+1}^{2 n} \mathcal{A}_{j k} \mathcal{B}_{i m} \delta_{m, k-n}\right) \\
& =\mathrm{i}\left(\sum_{k=1}^{n} \mathcal{A}_{j k} \mathcal{B}_{i, k+n}-\sum_{k=n+1}^{2 n} \mathcal{A}_{j k} \mathcal{B}_{i, k-n}\right) \\
& =\mathrm{i}\left(\sum_{k=1}^{n} \mathcal{A}_{j k} \mathcal{B}_{i, k+n}-\mathcal{A}_{j, k+n} \mathcal{B}_{i k}\right) \\
& =\mathrm{i}\left(\sum_{k=1}^{n}\left(\mathcal{A}_{a}\right)_{j k}\left(\mathcal{B}_{b}\right)_{i k}-\left(\mathcal{A}_{b}\right)_{j k}\left(\mathcal{B}_{a}\right)_{i k}\right) \\
& =\mathrm{i}\left(\mathcal{B}_{b} \mathcal{A}_{a}^{T}-\mathcal{B}_{a} \mathcal{A}_{b}^{T}\right)_{i j},
\end{aligned}
$$

which implies that

$$
|\operatorname{det} \mathbf{K}|=\left|\operatorname{det}\left(\mathcal{B}_{b} \mathcal{A}_{a}^{T}-\mathcal{B}_{a} \mathcal{A}_{b}^{T}\right)\right|
$$

as proven in [56]. Hence, equation (B.14) can finally be expressed as

$$
\operatorname{det} \boldsymbol{\Gamma}=\operatorname{det} \gamma|\operatorname{det} \mathbf{K}|^{2}
$$

that is, equation (75).

${ }^{13}(\cdot)^{-T}$ denotes the transpose of the inverse. 


\section{Appendix C. Pure Gaussian states as eigenvectors of $\frac{1}{2} R^{T} \Gamma^{-1} R$}

Here, we show that $n$-modal pure Gaussian states are eigenvectors of the operator $\frac{1}{2} \mathbf{R}^{T} \boldsymbol{\Gamma}^{-1} \mathbf{R}$ with eigenvalue $n$, see equation (91). In state space, a pure Gaussian state can be written as $\left|\psi^{\mathrm{G}}\right\rangle=\hat{S}|0\rangle$, where $\hat{S}$ is a Gaussian unitary and $|0\rangle$ is the $n$-modal vacuum state. Since the states considered in the proof of equation (71) are centered at the origin, we do not need to apply a displacement operator and $\hat{S}$ is a $n$-modal squeezing operator (with arbitrary squeezing and rotation). In order to apply $\frac{1}{2} \mathbf{R}^{T} \boldsymbol{\Gamma}^{-1} \mathbf{R}$ onto state $\left|\psi^{\mathrm{G}}\right\rangle$, we write the canonical transformation of $\mathbf{r}$ in phase space that corresponds to $\hat{S}$ in state space (in the Heisenberg picture), namely $\hat{S}^{\dagger} \mathbf{r} \hat{S}=\mathcal{M} \mathbf{r}$, where $\mathcal{M}$ is a symplectic matrix so that $\gamma^{\mathrm{G}}=\mathcal{M} \gamma_{\text {vac }} \mathcal{M}^{T}$. Remember that $\gamma^{\mathrm{G}}$ is the covariance matrix for the $x, p$-quadratures, but we are interested in the covariance matrix $\boldsymbol{\Gamma}$ for the $y, z$ quadratures. We thus use the following change of variables

$\hat{S}^{\dagger}\left(\begin{array}{l}\mathbf{y} \\ \mathbf{z}\end{array}\right) \hat{S}=\hat{S}^{\dagger}\left(\begin{array}{cc}\mathcal{A}_{a} & \mathcal{A}_{b} \\ \mathcal{B}_{a} & \mathcal{B}_{b}\end{array}\right)\left(\begin{array}{l}\mathbf{x} \\ \mathbf{p}\end{array}\right) \hat{S}=\left(\begin{array}{cc}\mathcal{A}_{a} & \mathcal{A}_{b} \\ \mathcal{B}_{a} & \mathcal{B}_{b}\end{array}\right) \hat{S}^{\dagger}\left(\begin{array}{l}\mathbf{x} \\ \mathbf{p}\end{array}\right) \hat{S}=\left(\begin{array}{cc}\mathcal{A}_{a} & \mathcal{A}_{b} \\ \mathcal{B}_{a} & \mathcal{B}_{b}\end{array}\right) \mathcal{M}\left(\begin{array}{l}\mathbf{x} \\ \mathbf{p}\end{array}\right)$

where we have use the fact that $\hat{S}$ and $\left(\begin{array}{ll}\mathcal{A}_{a} & \mathcal{A}_{b} \\ \mathcal{B}_{a} & \mathcal{B}_{b}\end{array}\right)$ commute since they act on two different spaces. Then, we have

$$
\begin{aligned}
& \frac{1}{2} \mathbf{R}^{T} \boldsymbol{\Gamma}^{-1} \mathbf{R}\left|\psi^{\mathrm{G}}\right\rangle=\frac{1}{2} \mathbf{R}^{T} \boldsymbol{\Gamma}^{-1} \mathbf{R} \hat{S}|0\rangle \\
& =\frac{1}{2} \hat{S} \hat{S}^{\dagger} \mathbf{R}^{T} \hat{S} \Gamma^{-1} \hat{S}^{\dagger} \mathbf{R} \hat{S}|0\rangle
\end{aligned}
$$

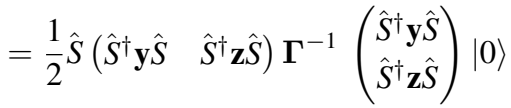

$$
\begin{aligned}
& =\frac{1}{2} \hat{S}\left(\begin{array}{ll}
\mathbf{x} & \mathbf{p}
\end{array}\right) \mathcal{M}^{T}\left(\begin{array}{cc}
\mathcal{A}_{a} & \mathcal{A}_{b} \\
\mathcal{B}_{a} & \mathcal{B}_{b}
\end{array}\right)^{T} \Gamma^{-1}\left(\begin{array}{cc}
\mathcal{A}_{a} & \mathcal{A}_{b} \\
\mathcal{B}_{a} & \mathcal{B}_{b}
\end{array}\right) \mathcal{M}\left(\begin{array}{l}
\mathbf{x} \\
\mathbf{p}
\end{array}\right)|0\rangle \\
& =\frac{1}{2} \hat{S} \mathbf{r}^{T} \gamma_{\text {vac }}^{-1} \mathbf{r}|0\rangle \\
& =\hat{S}\left(|\mathbf{x}|^{2}+|\mathbf{p}|^{2}\right)|0\rangle \\
& =n \hat{S}|0\rangle=n\left|\psi^{\mathrm{G}}\right\rangle \text {. }
\end{aligned}
$$

To find the fifth line, we have used equation (B.8) in order to compute the inverse of $\boldsymbol{\Gamma}$, namely

$\boldsymbol{\Gamma}^{-1}=\left[\left(\begin{array}{cc}\mathcal{A}_{a} & \mathcal{A}_{b} \\ \mathcal{B}_{a} & \mathcal{B}_{b}\end{array}\right) \gamma^{\mathrm{G}}\left(\begin{array}{cc}\mathcal{A}_{a} & \mathcal{A}_{b} \\ \mathcal{B}_{a} & \mathcal{B}_{b}\end{array}\right)^{T}\right]^{-1}=\left[\left(\begin{array}{cc}\mathcal{A}_{a} & \mathcal{A}_{b} \\ \mathcal{B}_{a} & \mathcal{B}_{b}\end{array}\right) \mathcal{M} \gamma_{\mathrm{vac}} \mathcal{M}^{T}\left(\begin{array}{cc}\mathcal{A}_{a} & \mathcal{A}_{b} \\ \mathcal{B}_{a} & \mathcal{B}_{b}\end{array}\right)^{T}\right]^{-1}$

Thus, equation (C.2) expresses that $\left|\psi^{\mathrm{G}}\right\rangle$ is an eigenvector of $\frac{1}{2} \mathbf{R}^{T} \boldsymbol{\Gamma}^{-1} \mathbf{R}$ with eigenvalue $n$, as advertized.

\section{ORCID iDs}

Anaelle Hertz (1) https://orcid.org/0000-0002-9608-512X 


\section{References}

[1] Heisenberg W 1927 Über den anschaulichen Inhalt der quantentheoretischen Kinematik und Mechanik Z. Phys. 43 172-98

[2] Kennard E H 1927 Zur Quantenmechanik einfacher Bewegungstypen Z. Phys. 44 326-52

[3] Schrödinger E 1932 Zum Heisenbergschen unschärfeprinzip Sitzber. Preuss. Akad. Wiss. 19 296-323

[4] Robertson H P 1930 A general formulation of the uncertainty principle and its classical interpretation Phys. Rev. 35667

[5] Hirschman I I 1957 A note on entropy Am. J. Math. 79 152-6

[6] Bialynicki-Birula I and Mycielski J 1975 Uncertainty relations for information entropy in wave mechanics Commun. Math. Phys. 44 129-32

[7] Beckner W 1975 Inequalities in Fourier analysis Ann. Math. 102 159-82

[8] Babenko K I 1961 An inequality in the theory of Fourier integrals Izv. Akad. Nauk SSSR Ser. Mat. 25 531-42

Babenko K I 1966 An inequality in the theory of Fourier integrals Am. Math. Soc. Transl. 44 11528 (Engl. transl.)

[9] Hertz A, Jabbour M G and Cerf N J 2017 Entropy-power uncertainty relations : towards a tight inequality for all Gaussian pure states J. Phys. A: Math. Theor. 50385301

[10] Renes J M and Boileau J-C 2009 Conjectured strong complementary information tradeoff Phys. Rev. Lett. 103020402

[11] Berta M, Christandl M, Colbeck R, Renes J M and Renner R 2010 The uncertainty principle in the presence of quantum memory Nat. Phys. 6 659-62

[12] Grosshans F and Cerf N J 2004 Continuous-variable quantum cryptography is secure against nonGaussian attacks Phys. Rev. Lett. 92047905

[13] Tomamichel M and Renner R 2011 The uncertainty relation for smooth entropies Phys. Rev. Lett. 106110506

[14] Tomamichel M, Lim C C W, Gisin N and Renner R 2010 Tight finite-key analysis for quantum cryptography Nat. Commun. 3634

[15] Furrer F, Franz T, Berta M, Leverrier A, Scholz V B, Tomamichel M and Werner R F 2012 Continuous variable quantum key distribution: finite-key analysis of composable security against coherent attacks Phys. Rev. Lett. 109100502

[16] Duan L M, Giedke G, Cirac J I and Zoller P 2000 Inseparability criterion for continuous variable systems Phys. Rev. Lett. 842722

[17] Simon R 2000 Peres-Horodecki separability criterion for continuous variable systems Phys. Rev. Lett. 842726

[18] Walborn S P, Taketani B G, Salles A, Toscano F and de Matos Filho R L 2009 Entropic entanglement criteria for continuous variables Phys. Rev. Lett. 103160505

[19] Huang Y 2013 Entanglement detection: complexity and Shannon entropic criteria IEEE Trans. Inf. Theory $598157-81$

[20] Rastegin A E 2017 Renyi formulation of entanglement criteria for continuous variables Phys. Rev. 95042334

[21] Hertz A, Karpov E, Mandilara A and Cerf N J 2016 Detection of non-Gaussian entangled states with an improved continuous-variable separability criterion Phys. Rev. A 93032330

[22] Deutsch D 1983 Uncertainty in quantum measurements Phys. Rev. Lett. 50631

[23] Kraus K 1987 Complementary observables and uncertainty relations Phys. Rev. D 353070

[24] Maassen H and Uffink J B M 1988 Generalized entropic uncertainty relations Phys. Rev. Lett. 60 1103

[25] Coles P J, Berta M, Tomamichel M and Wehner S 2017 Entropic uncertainty relations and their applications Rev. Mod. Phys. 8915002

[26] Bialynicki-Birula I and Rudnicki L 2011 Entropic uncertainty relations in quantum physics Statistical Complexity ed K Sen (Berlin: Springer) pp 1-34

[27] Toscano F, Tasca D S, Rusnicki L and Walborn S P 2018 Uncertainty relations for coarse-grained measurements: an overview Entropy 20454

[28] Robertson H P 1934 An indeterminacy relation for several observables and its classical interpretation Phys. Rev. 46794

[29] Kechrimparis S and Weigert S 2014 Heisenberg uncertainty relation for three canonical observables Phys. Rev. A 90062118 
[30] Dodonov V V 2018 Variance uncertainty relations without covariances for three and four observables Phys. Rev. A 97022105

[31] Kechrimparis S and Weigert S 2018 Geometry of uncertainty relations for linear combinations of position and momentum J. Phys. A: Math. Theor. 51025303

[32] Busch P, Heinonen T and Lahti P 2007 Heisenberg's uncertainty principle Phys. Rep. 452 155-76

[33] Ozawa M 2003 Universally valid reformulation of the Heisenberg uncertainty principle on noise and disturbance in measurement Phys. Rev. A 67042105

[34] Busch P, Lahti P and Werner R F 2013 Proof of Heisenberg's error-disturbance relation Phys. Rev. Lett. 111160405

[35] Busch P, Lahti P and Werner R F 2014 Heisenberg uncertainty for qubit measurements Phys. Rev. A 89012129

[36] Robertson H P 1929 The uncertainty principle Phys. Rev. 34163

[37] Weedbrook C, Pirandola S, Garcia-Patron R, Cerf N J, Ralph T C, Shapiro J H and Lloyd S 2012 Gaussian quantum information Rev. Mod. Phys. 84 621-69

[38] Simon R, Sudarshan E C G and Mukunda N 1987 Gaussian-Wigner distributions in quantum mechanics and optics Phys. Rev. A 363868

[39] Hertz A 2018 Exploring continuous-variable entropic uncertainty relations and separability criteria in quantum phase space $P h D$ Thesis Université libre de Bruxelles

[40] Cayley A 1849 Sur les déterminants gauches J. Reine Angew. Math. 38 93-6

[41] Trifonov D A 1997 Robertson intelligent states J. Phys. A: Math. Gen. 305941

[42] Cover T M and Thomas J A 2006 Elements of Information Theory (New York: Wiley)

[43] Beckner W 1975 Inequalities in Fourier analysis on $R^{n}$ Proc. Natl Acad. Sci. USA 72638

[44] Brascamp H J and Lieb E H 1976 Best constants in Young's inequality, its converse and its generalization to more than three functions Adv. Math. 20151

[45] Son W 2015 Role of quantum non-Gaussian distance in entropic uncertainty relations Phys. Rev. A 92012114

[46] Genoni M G, Paris M G A and Banaszek K 2008 Quantifying the non-Gaussian character of a quantum state by quantum relative entropy Phys. Rev. A 78060303

[47] Bialynicki-Birula I 2006 Formulation of the uncertainty relations in terms of the Rényi entropies Phys. Rev. A 74052101

[48] Jizba P, Ma Y, Hayes A and Dunningham J A 2016 One-parameter class of uncertainty relations based on entropy power Phys. Rev. E 93060104

[49] Bialynicki-Birula I 2007 Rényi entropy and the uncertainty relations AIP Conf. Proc. 88952

[50] Rastegin A E 2015 Uncertainty relations for general canonically conjugate observables in terms of unified entropies Found. Phys. 45 923-42

[51] Rudnicki L, Walborn S P and Toscano F 2012 Optimal uncertainty relations for extremely coarsegrained measurements Phys. Rev. A 85042115

[52] Jackiw R 1968 Minimum uncertainty product, number phase uncertainty product, and coherent states J. Math. Phys. 9339

[53] Hall M J W 1994 Noise-dependent uncertainty relations for the harmonic oscillator Phys. Rev. A 4942

[54] Guanlei X, Xiaotong W and Xiaotong X 2009 Generalized entropic uncertainty principle on fractional Fourier transform Signal Process. 892692

[55] Huang Y 2011 Entropic uncertainty relations in multidimensional position and momentum spaces Phys. Rev. A 83052124

[56] Hertz A, Vanbever L and Cerf N J 2018 Multidimensional entropic uncertainty relation based on a commutator matrix in position and momentum spaces Phys. Rev. A 97012111

[57] Guanlei X, Xiaotong W and Xiaotong X 2009 Uncertainty inequalities for linear canonical transform IET Signal Process. 3 392-402

[58] Bialynicki-Birula I 1985 Entropic uncertainty relations in quantum mechanics Quantum Probability and Applications II (Lecture Notes in Mathematics vol 1136) ed L Accardi and W von Waldenfels (Berlin: Springer) pp 90-103

[59] Hall M J W 1993 Phase resolution and coherent phase states J. Mod. Phys. 40 809-24

[60] Hall M J W 2008 Almost-periodic time observables for bound quantum systems J. Phys. A: Math. Theor. 41255301

[61] Coles P J, Katariya V, Marvian I and Wilde M M 2018 Entropic energy-time uncertainty relation (arXiv:1805.07772 [quant-ph]) 
[62] Furrer F, Berta M, Tomamichel M, Scholz V B and Christandl M 2014 Position-momentum uncertainty relations in the presence of quantum memory J. Math. Phys. 55122205

[63] Rastegin A E 2016 Entropic uncertainty relations for successive measurements of canonically conjugate observables Ann. Phys. 528 835-44

[64] Magnus J R and Neudecker H 1988 Matrix Differential Calculus with Applications in Statistics and Econometrics (New York: Wiley)

[65] Hertz A, Oreshkov O and Cerf N J 2019 (in preparation)

[66] Simon R, Mukunda N and Dutta B 1994 Quantum-noise matrix for multimode systems: U(n) invariance, squeezing, and normal forms Phys. Rev. A 491567

[67] Williamson J 1936 On the algebraic problem concerning the normal forms of linear dynamical systems Am. J. Math. 58 141-63

[68] Serafini A, Illuminati F and De Siena S 2003 Symplectic invariants, entropic measures and correlations of Gaussian states J. Phys. B: At. Mol. Opt. Phys. 37 L21

[69] Silvester J 2000 Determinants of block matrices Math. Gaz. 84 460-7 